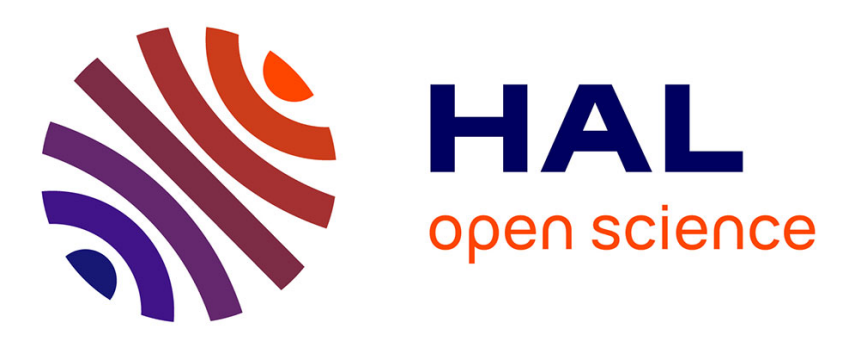

\title{
Numerical validation of an Homogenized Interface Model
}

Giuseppe Geymonat, Sofiane Hendili, Françoise Krasucki, Marina Vidrascu

\section{To cite this version:}

Giuseppe Geymonat, Sofiane Hendili, Françoise Krasucki, Marina Vidrascu. Numerical validation of an Homogenized Interface Model. Computer Methods in Applied Mechanics and Engineering, 2014, 269, pp.356-380. 10.1016/j.cma.2013.11.009 . hal-00839616v2

\section{HAL Id: hal-00839616 https://hal.inria.fr/hal-00839616v2}

Submitted on 3 Dec 2013

HAL is a multi-disciplinary open access archive for the deposit and dissemination of scientific research documents, whether they are published or not. The documents may come from teaching and research institutions in France or abroad, or from public or private research centers.
L'archive ouverte pluridisciplinaire HAL, est destinée au dépôt et à la diffusion de documents scientifiques de niveau recherche, publiés ou non, émanant des établissements d'enseignement et de recherche français ou étrangers, des laboratoires publics ou privés. 


\title{
Numerical validation of an Homogenized Interface Model $^{\text {th }}$
}

\author{
Giuseppe Geymonat ${ }^{\mathrm{a}}$, Sofiane Hendilib ${ }^{\mathrm{b}}$, Françoise Krasucki ${ }^{\mathrm{c}}$, Marina \\ Vidrascu $^{\text {d,e }}$ \\ ${ }^{a}$ LMS, UMR-CNRS 7649, Ecole Polytechnique,Route de Saclay 91128 Palaiseau Cedex \\ ${ }^{b} C E A, D E N$, DANS, DM2S, SEMT, Laboratoire de Modélisation et de Simulation des \\ Structures, 91191 Gif-sur-Yvette, France \\ ${ }^{c}$ I3M, UMR-CNRS 5149, Université Montpellier 2, Case courier 051, Place Eugène \\ Bataillon, 34095 Montpellier Cedex 5 \\ ${ }^{d}$ Inria, REO project-team, Rocquencourt - B.P. 105, F-78153 Le Chesnay cedex, France \\ ${ }^{e} U P M C$ Univ Paris VI, REO project-team, UMR 7958 LJLL, F-75005 Paris, France
}

\begin{abstract}
The aim of this paper is to numerically validate the effectiveness of a matched asymptotic expansion formal method introduced in a pioneering paper by Nguetseng and Sànchez Palencia [1] and extended in [2], [3]. Using this method a simplified model for the influence of small identical heterogeneities periodically distributed on an internal surface to the overall response of a linearly elastic body is derived. In order to validate this formal method a careful numerical study compares the solution obtained by a standard method on a fine mesh to the one obtained by asymptotic expansion. We compute both the zero and the first order terms in the expansion. To efficiently compute the first order term we introduce a suitable domain decomposition method.
\end{abstract}

Keywords: finite element method, linear elasticity, matched asymptotic expansions, domain decomposition

\footnotetext{
This work was partially supported by the French Agence Nationale de la Recherche (ANR) under Grant Epsilon (BLAN08-2 312370) (Domain decomposition and multi-scale computations of singularities in mechanical structures); it is a natural continuation of the Thesis of Sofiane Hendili carried out at I3M and INRIA and supported by Grant Epsilon.

Email addresses: giuseppe.geymonat@lms .polytechnique.fr (Giuseppe Geymonat), s_hendili@yahoo.fr (Sofiane Hendili), krasucki@math.univ-montp2.fr (Françoise Krasucki), marina.vidrascu@inria.fr ( Marina Vidrascu)
} 


\section{Contents}

1 Introduction 2

2 Asymptotic model 5

2.1 The problem . . . . . . . . . . . . . . 5

2.2 The multi-scale inner-outer matched asymptotic method . . 7

2.2.1 Theoretical results for the asymptotic study . . . . . . 12

2.3 Definition of the outer and inner approximations . . . . . . . 17

3 Solution of the first order problem by domain decomposition 17

3.1 A linear elasticity model problem . . . . . . . . . . . . 17

3.2 Solution of the first order problem . . . . . . . . . . . . . 19

3.3 Implementation issues . . . . . . . . . . . . . . . . . . 21

4 Validation 23

4.1 Description of the test problem . . . . . . . . . . . 24

4.2 Numerical solution of the cell problems . . . . . . . . . 25

4.2.1 Dependence on $L^{\infty} \ldots \ldots \ldots \ldots$

4.2 .2 Dependence on $f \ldots \ldots \ldots \ldots \ldots$

4.2 .3 Dependence on $d \ldots \ldots \ldots \ldots$. . . . . . . . . . . 28

4.2.4 Dependence on $E^{I} / E$ in the case of elastic inclusions with $p=0 \ldots \ldots \ldots \ldots \ldots \ldots$

4.3 Validation at the macroscopic scale . . . . . . . . . . 32

4.4 Validation at the microscopic scale: the domain $\Omega_{h}^{i n}(\varepsilon) \ldots 35$

4.5 Validation at the microscopic scale: the stress field near the inclusions . . . . . . . . . . . . . . . 39

5 Concluding remarks $\quad 39$

\section{Introduction}

For various researches and applications in fields spanning the mathematical, physical and engineering communities, there is great interest in efficient and mathematically rigorously justified numerical approximations of the solutions of boundary value problems where the domain and/or the coefficients 
have a large number of heterogeneities. Several such situations were investigated:

i) The heterogeneities have a characteristic size which is a power of $\varepsilon$ and are periodically distributed in the whole volume; the ratio between the size of the period and the size of the entire structure is traditionally denoted $\varepsilon$. In order to obtain numerically efficient (i.e. precise and not too computationally demanding) simplified models it is now classical to use an homogenization method. An advantage of the homogenization methods is that they have been developed and fully mathematically justified for many different geometrical and/or mechanical circumstances, in particular when the ratio between the mechanical characteristics of the heterogeneities and of the surrounding material can depend on $\varepsilon$ [4], [5], [6], [7].

ii) Another situation of interest for the engineering community arises when two bodies are pasted together using a thin layer of another medium whose transversal depth has a ratio $\varepsilon$ to the whole structure. Many numerically efficient simplified models have been constructed and mathematically fully justified for various mechanical and /or geometrical properties (see e.g. [8], [9], [10] [11]).

iii) A situation that is new, even if there are similarities with the two previous ones, arises when the thin layer is generated by heterogeneities with characteristic size of order $\varepsilon$. These heterogeneities are periodically distributed, with period $\varepsilon$, on an internal surface $\omega$. This case is considered in the present work.

It is obvious that in this case the use of standard finite element methods rapidly becomes very expensive and thus it is mandatory to find numerically efficient and, if possible, mathematically justified models to find both global (i.e. on the whole structure) and local (i.e near the heterogeneities) behavior. For heterogeneities which are holes, Nguetseng and Sànchez Palencia have proposed, for the local behavior, in a pioneering research, [1] to use a formal multi-scale matched asymptotic expansion. The initial problem is replaced by a set of new ones for which the layer of heterogeneities becomes a surface $\omega$ on which particular non-homogeneous jump conditions are defined. In the recent years this formal method has been used again,mainly for the global behavior, in different geometrical situations and has been generalized in order to consider not only holes but also elastic inclusions [12], [13], [14], [15].

The first objective of this paper is to numerically validate a recently proposed variant of the multi-scale matched asymptotic expansion method [2], 
[3] that can be successfully applied both for the global and the local behavior. In Section 2, we recall this formal method adapted to the case where different types of heterogeneities are considered. Specifically we assume that the displacement and stress fields admit two asymptotic expansions, one far from the heterogeneities (the outer one) another one close to them (the inner one). The formal construction of the outer and inner problems allows to define the transmission coefficients across the surface. As in [1] we show that the order 0 outer problem is independent of the heterogeneities. For the first order outer problem, that was not studied in [1], since the authors were interested by a local behavior, the transmission coefficients are given by several elementary inner problems posed at the so-called microscopic level, i.e. on a representative cell. These coefficients depend on the geometry and the nature of the heterogeneities (holes, highly contrasted materials). The outer problems are usually called the macroscopic problems. The numerical validation is described in Section 4 on a 2D test problem. The objective of this numerical validation is to study, in an appropriate norm, the error between the reference solution and the numerical solution obtained by asymptotic expansions both for zero order and first order. Since the exact solution is not known the reference solution is the numerical solution of the original problem obtained using standard finite elements on a very fine mesh. The mesh-size $h$ has to be fine enough so that the approximation error be negligible. It is essential to study the evolution of this error when $\varepsilon$ decreases. In Section 4.2 we have evaluated, for the coefficients appearing in the transmission conditions, the sensitivity with respect to the different parameters of the cell problems used to compute them.

The second objective is to give an efficient numerical algorithm which implements the computation of the macroscopic outer approximation obtained trough the zero and the first order terms. Thus in Section 3 we introduce a domain decomposition type algorithm to numerically solve the first order problem. The novelty of the situation is due to the fact that on the surface $\omega$ one has non-homogeneous transmission conditions both for the displacements and for the normal stresses.

We conclude the numerical validation in Section 4.5 with the comparison of the distribution of the stresses around the heterogeneities in the case of holes and of elastic inclusions. This comparison demonstrates the numerical efficiency for the local behavior, of our variant [2], [3] of the multi-scale matched asymptotic expansion method. 


\section{Asymptotic model}

\subsection{The problem}

Let us consider a three dimensional structure $\Omega$ (an open domain of $\mathbb{R}^{3}$ with smooth boundary $\partial \Omega$ ) containing small identical heterogeneities periodically distributed along a surface $\omega$ that we assume for simplicity contained in the plane $x_{1}=0$ (see Fig. 1). We assume that $\omega$ is contained in the union of

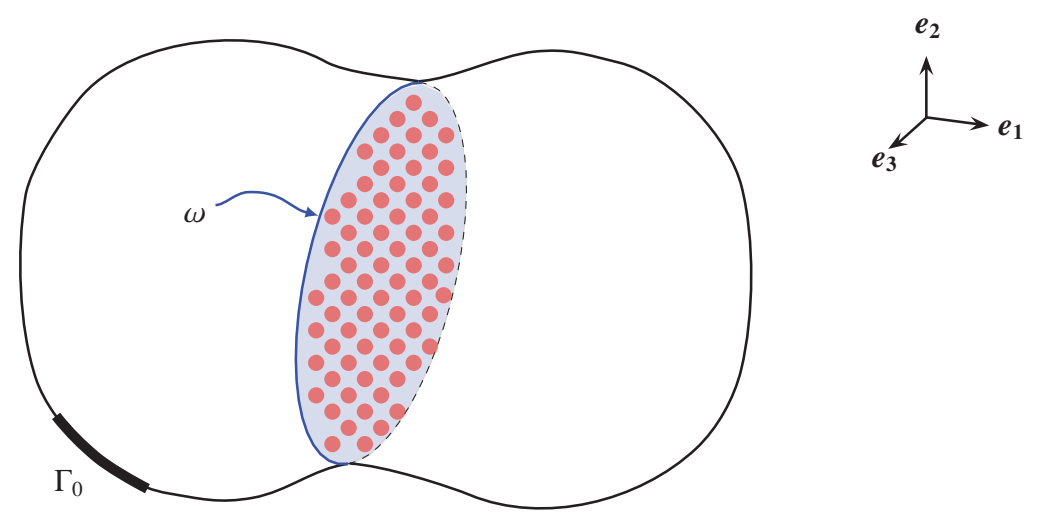

Figure 1: The structure with the layer of heterogeneities

$\mathcal{N}(\varepsilon) \approx \frac{\operatorname{area}(\omega)}{\operatorname{area}(\hat{Y})} \varepsilon^{-2}$ sets $\varepsilon \hat{Y}$, where $\hat{Y} \subset \mathbb{R}^{2}$ is the basis of a periodic planar net (we take for simplicity $\hat{Y}=]-\frac{1}{2}, \frac{1}{2}[\times]-\frac{1}{2}, \frac{1}{2}[$ ). Let $I$ be a non-empty $3 D$ domain contained in $\mathbb{R} \times \hat{Y}$ with smooth boundary $\partial I$, with diameter $d$ satisfying $0<\frac{d}{\operatorname{diam}(\Omega)}<+\infty$ and such that $I \cap\left\{x_{1}=0\right\}$ is strictly contained in $\hat{Y}$ (hence $d<\sqrt{2}$ ). We also assume that the heterogeneities have a diameter $\varepsilon d$ and fill every domain $\varepsilon I$. We denote by $\varepsilon \partial I$ the boundary of $\varepsilon I$ and by $\mathcal{I}^{\varepsilon}$, resp. $\partial \mathcal{I}^{\varepsilon}$, the union of all the heterogeneities, resp. of all their boundaries.

Hence from a geometrical point of view there are two natural length scales: the first is a global one (the $3 D$-diameter of $\Omega$ or the $2 D$-diameter of $\omega$ ) the other one is a local one connected with the heterogeneities (e.g. the diameter of each heterogeneity). The ratio between these two scales will be denoted by $\varepsilon$. More precisely, the parameter $\varepsilon$ is a non-dimensional parameter characterizing the geometrical distribution of the heterogeneities in the structure 
since, at the same time, it characterizes the ratio between the diameter of any heterogeneity $(\varepsilon d)$ and the diameter of $\Omega$ and the ratio between the diameter of the period $(\varepsilon \hat{Y})$ of the planar net and the diameter of the planar set $\omega$ supporting the heterogeneities.

As in many homogenization situations, in this work we consider two types of heterogeneities: holes and elastic inclusions with stiffness dependent on a power of $\varepsilon$. In the case of elastic inclusions, transmission conditions must be verified on $\partial \mathcal{I}^{\varepsilon}$, while free boundary conditions must be taken into account on $\partial \mathcal{I}^{\varepsilon}$ in the case of the holes. The boundary of $\Omega$ is composed of two parts $\Gamma_{0}$ and $\Gamma_{F}$ with $\partial \Omega=\overline{\Gamma_{0}} \cup \overline{\Gamma_{F}}$ and $\Gamma_{0} \cap \Gamma_{F}=\emptyset$. The structure is clamped on $\Gamma_{0}$ and a density of surface forces $\boldsymbol{F}$ is applied on the complementary part $\Gamma_{F}$ (in order to avoid inessential technicalities we assume null volume forces). Let $\boldsymbol{u}^{\varepsilon}$ and $\boldsymbol{\sigma}^{\varepsilon}$ be the displacement and the stress field at equilibrium. These fields verify the following equations:

$$
\begin{cases}\boldsymbol{d i v} \boldsymbol{\sigma}^{\varepsilon}=\mathbf{0} & \text { in } \Omega^{\varepsilon} \\ \boldsymbol{\sigma}^{\varepsilon}=\boldsymbol{A} \boldsymbol{\gamma}\left(\boldsymbol{u}^{\varepsilon}\right) & \text { in } \Omega^{\varepsilon} \\ \boldsymbol{\sigma}^{\varepsilon} \boldsymbol{n}=\boldsymbol{F} & \text { on } \Gamma_{F} \\ \boldsymbol{u}^{\varepsilon}=\mathbf{0} & \text { on } \Gamma_{0}\end{cases}
$$

where

$$
\Omega^{\varepsilon}= \begin{cases}\Omega \backslash \mathcal{I}^{\varepsilon} & \text { in the holes case } \\ \Omega & \text { in the elastic inclusions case }\end{cases}
$$

$$
\boldsymbol{A}= \begin{cases}\boldsymbol{A}^{S} & \text { in } \Omega \backslash \mathcal{I}^{\varepsilon} \\ \varepsilon^{p} \boldsymbol{A}^{I}, p \in \mathbb{N} & \text { in } \mathcal{I}^{\varepsilon} \text { (in the case of elastic inclusions) }\end{cases}
$$

where the linear elasticity tensors $A^{S}$ and $A^{I}$ have the same magnitude.

Let us explicitly remark that we consider only linearly elastic materials and that the elasticity tensors $A^{S}$ and $A^{I}$ satisfy the usual properties of symmetry and positive definiteness. Let us stress that in the case of elastic inclusions as shown in (2) the parameter $\varepsilon$ is a non-dimensional parameter characterizing at the same time the geometrical distribution of the heterogeneities in the 
structure and the ratio between the rigidity of the heterogeneities and the rigidity of the structure.

Remark 1: In the applications, the choice of the value of $p$ in equation (2)s is very important. $p=0$ is clearly perfectly adapted when the elastic coefficients of the inclusions $\boldsymbol{A}^{I}$ are of the same order of magnitude than the elastic coefficients of the surrounding material $\boldsymbol{A}^{S}$. In the case of weak heterogeneities i.e. when the coefficients $\boldsymbol{A}^{S}$ are much more greater than those of the heterogeneities ; the choice $p>0$ is more appropriate. An interesting consequence of the model obtained with the subsequent analysis is that up to the first order approximation weak inclusions (i.e. with $p>0$ ) mainly behave as holes.

\subsection{The multi-scale inner-outer matched asymptotic method}

The inner-outer matched asymptotic method approximates the initial problem with a set of new ones defined in $\Omega$ where the layer of heterogeneities is replaced by the surface $\omega$ (see Fig. 2) where suitable transmission conditions are defined.

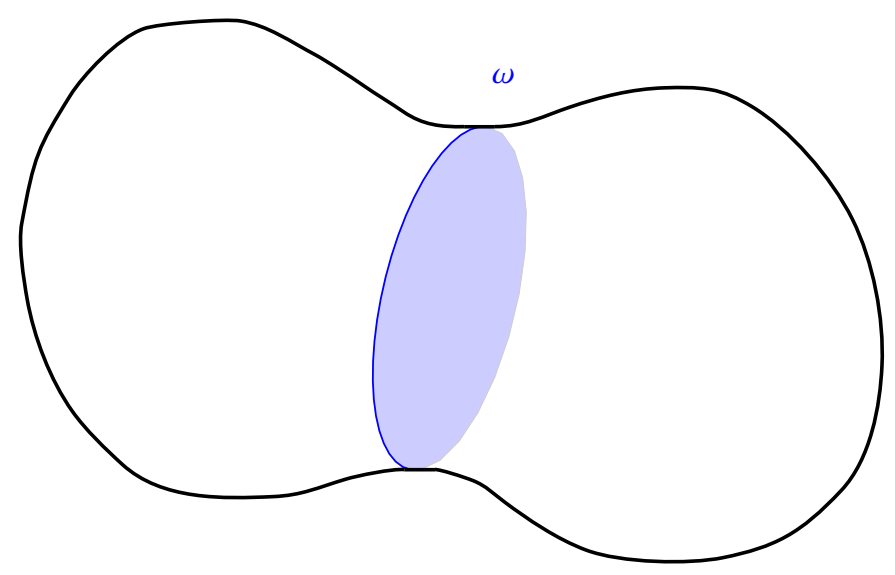

Figure 2: The structure without the layer of heterogeneities

The presence of the heterogeneities involves a boundary layer effect, i.e. a significant variation of the solution near $\omega$. Hence one has to use a matched asymptotic expansion for the normal variable to $\omega$ and a multi-scale method in order to track the homogenization in the tangential variables. More 
precisely the formal matched asymptotic expansion method applied to the present problem has the following steps:

1. Decomposition of the domain $\Omega$ into two overlapping subdomains. This decomposition depends on the choice of a function $\eta(\varepsilon)$ which has to satisfy the following mainly qualitative conditions:

$$
\left\{\begin{array}{l}
0<\varepsilon<\eta(\varepsilon) \\
\lim _{\varepsilon \rightarrow 0} \eta(\varepsilon)=0 \\
\lim _{\varepsilon \rightarrow 0} \frac{\eta(\varepsilon)}{\varepsilon}=+\infty
\end{array}\right.
$$

Using this function $\eta(\varepsilon)$ the overlapping domains $\Omega^{\text {out, } \varepsilon}$ and $\Omega^{i n, \varepsilon}$ are defined by (see Fig. 3 and Fig. 4) :

$\star \Omega^{\text {out }, \varepsilon}=\left\{\boldsymbol{x}=\left(x_{1}, x_{2}, x_{3}\right) \in \Omega ;\left|x_{1}\right|>\frac{\varepsilon}{2}\right\}$,

$\star \Omega^{i n, \varepsilon}=\left\{\boldsymbol{x}=\left(x_{1}, x_{2}, x_{3}\right) \in \Omega^{\varepsilon} ;\left|x_{1}\right|<\frac{\eta(\varepsilon)}{2}\right\}$

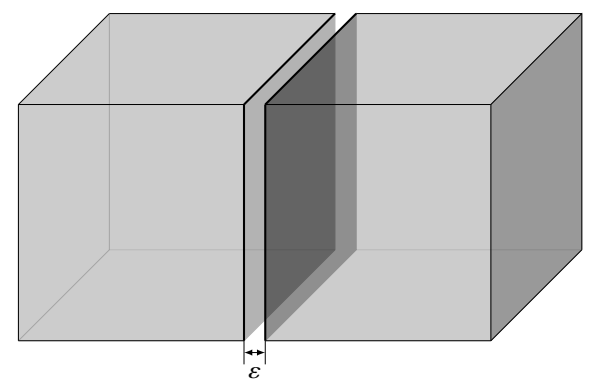

Figure 3: $\Omega^{\text {out }, \varepsilon}=\Omega^{+, \varepsilon} \cup \Omega^{-, \varepsilon}$

2. Introduction of the normalized coordinate systems.

$\star$ When $\varepsilon \rightarrow 0$, the domain $\Omega^{\text {out, } \varepsilon}$ tends to a fixed domain $\Omega^{-} \cup \Omega^{+}=$ $\Omega \backslash \omega=\left\{\boldsymbol{x} \in \Omega ;\left|x_{1}\right|>0\right\}$ which is also called the outer domain. The outer system of coordinates is $\boldsymbol{x}:=\left(x_{1}, x_{2}, x_{3}\right) \in \Omega \backslash \omega$.

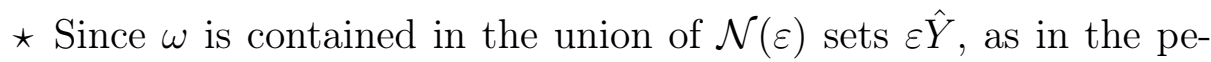
riodic homogenisation procedure the position $\boldsymbol{z}:=\left(z_{1}, z_{2}, z_{3}\right)$ of a point $M$ in the domain $\Omega^{i n, \varepsilon}$ can be given by two independent 


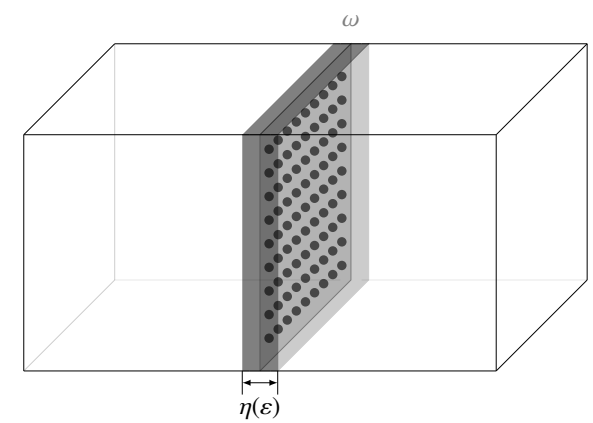

Figure 4: $\Omega^{i n, \varepsilon}$

data:

i) the macroscopic position $(0, \hat{\boldsymbol{x}}):=\left(0, x_{2}, x_{3}\right) \in \omega$ of the center of the period $\varepsilon \hat{Y}$ containing the projection of $M$ on $\omega$ ii) the microscopic position defined as the position of the point $M$ with respect to this center. To obtain a domain and a coordinate system independent from $\varepsilon$, one applies a dilatation of the variables $\varepsilon y_{i}$ of a factor $\frac{1}{\varepsilon}$. Using (3) and the periodicity assumption on the heterogeneities, the inner domain is $\omega \times Y$ where $Y:=\mathbb{R} \times \hat{Y}$ is called the basic cell (see Fig.5) and the relation between the coordinates $\boldsymbol{z}$ of the point $M$ and the point $((0, \hat{\boldsymbol{x}}), \boldsymbol{y})$ of the inner domain is given by:

$$
\boldsymbol{z}=(0, \hat{\boldsymbol{x}})+\varepsilon \boldsymbol{y}
$$

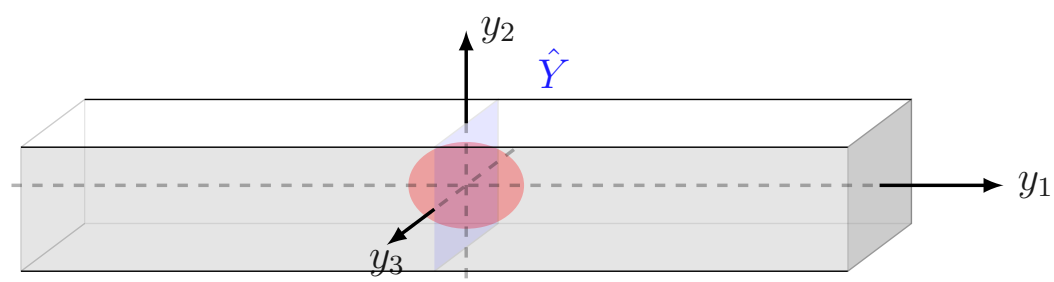

Figure 5: The basic cell $Y$

This correspondence implies that the operators divergence $(\boldsymbol{d i v})$ and symmetric gradient $(\gamma)$ must be reformulated in terms of the macroscopic $\hat{\boldsymbol{x}}$ and the microscopic $\boldsymbol{y}=\left(y_{1}, \hat{\boldsymbol{y}}\right)$ coordinates in the 
following way:

$$
\begin{array}{r}
\boldsymbol{d i v} \boldsymbol{\tau}(\hat{\boldsymbol{x}}, \boldsymbol{y})=\boldsymbol{d i v}_{x} \boldsymbol{\tau}(\hat{\boldsymbol{x}}, \boldsymbol{y})+\frac{1}{\varepsilon} \boldsymbol{d i v}_{y} \boldsymbol{\tau}(\hat{\boldsymbol{x}}, \boldsymbol{y}) \\
\gamma(\boldsymbol{v}(\hat{\boldsymbol{x}}, \boldsymbol{y}))=\gamma_{\boldsymbol{x}}(\boldsymbol{v}(\hat{\boldsymbol{x}}, \boldsymbol{y}))+\frac{1}{\varepsilon} \gamma_{\boldsymbol{y}}(\boldsymbol{v}(\hat{\boldsymbol{x}}, \boldsymbol{y}))
\end{array}
$$

with:

$$
\begin{cases}\boldsymbol{d i v}_{x} \boldsymbol{\tau}=\frac{\partial \tau_{i j}}{\partial x_{j}} \boldsymbol{e}_{i} & , \quad \boldsymbol{d i v}_{y} \boldsymbol{\tau}=\frac{\partial \tau_{i j}}{\partial y_{j}} \boldsymbol{e}_{i} \\ \left(\boldsymbol{\gamma}_{x}(\boldsymbol{v})\right)_{i j}=\frac{1}{2}\left(\frac{\partial v_{j}}{\partial x_{i}}+\frac{\partial v_{i}}{\partial x_{j}}\right) & , \quad\left(\boldsymbol{\gamma}_{y}(\boldsymbol{v})\right)_{i j}=\frac{1}{2}\left(\frac{\partial v_{j}}{\partial y_{i}}+\frac{\partial v_{i}}{\partial y_{j}}\right)\end{cases}
$$

3. Ansatz. A priori introduce two formal asymptotic expansions of $\boldsymbol{u}^{\varepsilon}$ :

* the outer expansion

$$
\boldsymbol{u}^{\varepsilon}\left(x_{1}, x_{2}, x_{3}\right)=\sum_{i=0}^{\infty} \varepsilon^{i} \boldsymbol{u}^{i}\left(x_{1}, x_{2}, x_{3}\right)
$$

* the inner expansion

$$
\boldsymbol{u}^{\varepsilon}\left(x_{1}, x_{2}, x_{3}\right)=\sum_{i=0}^{\infty} \varepsilon^{i} \boldsymbol{v}^{i}\left(\hat{\boldsymbol{x}}, y_{1}, \hat{\boldsymbol{y}}\right)
$$

where the $\boldsymbol{v}^{i}$ are $\hat{Y}$-periodic, i.e. such that:

$$
\boldsymbol{v}^{i}\left(\hat{\boldsymbol{x}}, y_{1}, \hat{\boldsymbol{y}}+p \boldsymbol{e}_{2}+q \boldsymbol{e}_{3}\right)=\boldsymbol{v}^{i}\left(\hat{\boldsymbol{x}}, y_{1}, \hat{\boldsymbol{y}}\right) \quad \forall(p, q) \in \mathbb{Z}^{2}
$$

and $\hat{\boldsymbol{x}} \in \omega$ has to be considered as a parameter.

Let us notice that in this context there are no convergence results for the formal series (7) and (8).

4. Deduction of the outer and inner expansion of $\boldsymbol{\sigma}^{\varepsilon}$. We use the constitutive equations and the inner and outer expansion of $\boldsymbol{u}^{\varepsilon}$ to determinate the outer and the inner expansion of $\boldsymbol{\sigma}^{\varepsilon}$.There is a difference between the case of holes and the case of elastic inclusions characterized by the elastic fourth-order tensor $\boldsymbol{A}^{\varepsilon, I}=\varepsilon^{p} \boldsymbol{A}^{I}$ with $\boldsymbol{A}^{I} \simeq \boldsymbol{A}$ and $p \in \mathbb{N}$. 
* Outer expansion. Outside the heterogeneities the structure is constituted by a linearly elastic material whose rigidity tensor is $\boldsymbol{A}$, and hence:

$$
\boldsymbol{\sigma}^{\varepsilon}(\boldsymbol{x})=\sum_{i=0}^{\infty} \varepsilon^{i} \boldsymbol{\sigma}^{i}(\boldsymbol{x})
$$

with

$$
\boldsymbol{\sigma}^{i}=\boldsymbol{A} \boldsymbol{\gamma}_{x}\left(\boldsymbol{u}^{i}\right)
$$

$\star$ Inner expansion. The distinction between the holes and the elastic inclusions is significant:

- in $Y \backslash I$ (for holes or elastic inclusions):

$$
\boldsymbol{\sigma}^{\varepsilon}(\boldsymbol{x})=\sum_{i=-1}^{\infty} \varepsilon^{i} \boldsymbol{\tau}^{i}(\hat{\boldsymbol{x}}, \boldsymbol{y})
$$

with

$$
\left\{\begin{array}{l}
\boldsymbol{\tau}^{-1}=\boldsymbol{A} \boldsymbol{\gamma}_{y}\left(\boldsymbol{v}^{0}\right) \\
\boldsymbol{\tau}^{i}=\boldsymbol{A} \boldsymbol{\gamma}_{x}\left(\boldsymbol{v}^{i}\right)+\boldsymbol{A} \boldsymbol{\gamma}_{y}\left(\boldsymbol{v}^{i+1}\right) \text { for } \quad i \geq 0
\end{array}\right.
$$

- in $I$ (only for elastic inclusions):

$$
\boldsymbol{\sigma}^{\varepsilon}(\boldsymbol{x})=\sum_{i=p-1}^{\infty} \varepsilon^{i} \boldsymbol{\tau}^{i}(\hat{\boldsymbol{x}}, \boldsymbol{y})
$$

with

$$
\left\{\begin{array}{l}
\boldsymbol{\tau}^{p-1}=\boldsymbol{A}^{I} \boldsymbol{\gamma}_{y}\left(\boldsymbol{v}^{0}\right) \\
\boldsymbol{\tau}^{i}=\boldsymbol{A}^{I} \boldsymbol{\gamma}_{x}\left(\boldsymbol{v}^{i-p}\right)+\boldsymbol{A}^{I} \boldsymbol{\gamma}_{y}\left(\boldsymbol{v}^{i-p+1}\right) \text { for } i \geq p
\end{array}\right.
$$

The fields $\boldsymbol{\tau}^{i}$ are $\hat{Y}$-periodic, with respect to $\hat{\boldsymbol{y}}$ and $\hat{\boldsymbol{x}} \in \omega$ has to be considered as a parameter.

5. Deduction of the boundary value problems verified by the different terms of the inner and outer expansion. In general the boundary value problems for the terms of the outer expansion need transmission conditions 
on $\omega$ that are not directly determined at this stage. The boundary value problems for the terms of the inner expansion may even have a non unique solution. Matching conditions are applied in order to complete the statement of the transmission conditions on $\omega$ and to completely determine each term of the inner formal asymptotic expansion.

The terms of each formal asymptotic expansion can be computed following these steps; in the sequel only the first two deserve attention. Let us explicitly remark that in the inner expansion the development of $\boldsymbol{\sigma}^{\varepsilon}$ begins with the term $\boldsymbol{\tau}^{-1}$ in $Y \backslash I$ and the term $\boldsymbol{\tau}^{p-1}$ in $I$.

\subsubsection{Theoretical results for the asymptotic study}

This section is a reminder without any proofs of the main results of the asymptotic study (see [2] for details).

Zeroth-order approximation: .

- inner approximation: the solution $\boldsymbol{v}^{0}$ verify

$$
\boldsymbol{v}^{0}(\boldsymbol{x}, \boldsymbol{y})=\boldsymbol{v}^{0}(\boldsymbol{x})=\boldsymbol{u}^{0}(0, \hat{\boldsymbol{x}})
$$

where $\boldsymbol{u}^{0}$ is the first term of the outer approximation

- outer approximation: $\boldsymbol{u}^{0}$ is solution of

$$
\begin{cases}\boldsymbol{d i v} \boldsymbol{\sigma}^{0}\left(\boldsymbol{u}^{0}\right)=\mathbf{0} & \text { in } \Omega_{0} \\ \boldsymbol{\sigma}^{0}=\boldsymbol{A} \boldsymbol{\gamma}\left(\boldsymbol{u}^{0}\right) & \text { in } \Omega_{0} \\ \boldsymbol{\sigma}^{0} \boldsymbol{n}=\boldsymbol{F} & \text { on } \Gamma_{F} \\ \boldsymbol{u}^{0}=\mathbf{0} & \text { on } \Gamma_{0}\end{cases}
$$

Remark 2: Notice that there are no jumps on $\omega$ for the zero order outer approximation. In other words, at the zero order the outer approximation does not take into account the heterogeneities. Thus this problem can be solved using a standard finite element procedure.

Remark 3: Assuming $\boldsymbol{A}$ constant (or regular) and $\boldsymbol{F}$ enough regular, the classical regularity results for linear elasticity [5] imply that the solution $\boldsymbol{u}^{0}$ is regular in $\bar{\Omega} \backslash\left(\overline{\Gamma_{0}} \cap \overline{\Gamma_{F}}\right)$ and hence on $\bar{\omega}$ when $\partial \omega \cap\left(\overline{\Gamma_{0}} \cap \overline{\Gamma_{F}}\right)=\emptyset$. 
First-order approximation: .

- inner approximation:

* The definition of $\boldsymbol{v}^{1}(\hat{\boldsymbol{x}}, \boldsymbol{y})$ is similar in the case of holes and in the case of elastic inclusions

$$
\boldsymbol{v}^{1}(\hat{\boldsymbol{x}}, \boldsymbol{y})=f\left(y_{1} ; a, b\right) \frac{\partial \boldsymbol{u}^{0}}{\partial x_{1}}(0, \hat{\boldsymbol{x}})+\frac{\partial u_{i}^{0}}{\partial x_{j}}(0, \hat{\boldsymbol{x}}) \boldsymbol{V}^{i j}(\boldsymbol{y})+\check{\boldsymbol{v}}(\hat{\boldsymbol{x}})
$$

where: $f\left(y_{1} ; a, b\right)$ is an odd function of class $\mathcal{C}^{2}(\mathbb{R})$ such that

$$
f\left(y_{1} ; a, b\right)= \begin{cases}0 & \text { if } 0<\left|y_{1}\right| \leq a \\ y_{1} & \text { if }\left|y_{1}\right| \geq b\end{cases}
$$

with $d<2 a<2 b$ (in the sequel, except when we wish to stress the dependence on $a, b$, we will simply write $\left.f\left(y_{1}\right)\right)$. Let us only stress that in the case of holes $\boldsymbol{y} \in Y \backslash I$ and in the case of elastic inclusions $\boldsymbol{y} \in Y$.

* When $\boldsymbol{y} \in Y \backslash I$ the definition of $\boldsymbol{\tau}^{0}$ is the same for holes and for elastic inclusions and follows from (13) :

$$
\boldsymbol{\tau}^{0}(\hat{\boldsymbol{x}}, \boldsymbol{y})=\boldsymbol{G}\left(y_{1}, \boldsymbol{u}^{0}\right)+\frac{\partial u_{i}^{0}}{\partial x_{j}}(0, \hat{\boldsymbol{x}}) \boldsymbol{A} \boldsymbol{\gamma}_{y}\left(\boldsymbol{V}^{i j}\right)(\boldsymbol{y})
$$

where the tensor $\boldsymbol{G}$ is defined by

$$
\boldsymbol{G}\left(y_{1}, \boldsymbol{u}^{0}\right)=\boldsymbol{A}\left(\boldsymbol{\gamma}_{\boldsymbol{x}}\left(\boldsymbol{u}^{0}(0, \hat{\boldsymbol{x}})\right)+f^{\prime}\left(y_{1}\right) \frac{\partial \boldsymbol{u}^{0}}{\partial x_{1}}(0, \hat{\boldsymbol{x}}) \otimes_{S} \boldsymbol{e}_{1}\right)
$$

* When $\boldsymbol{y} \in I$ then (14) and (15) imply that the definition of $\boldsymbol{\tau}^{0}$ depends on $p$. More precisely when $p \geq 1$ one has $\tau^{0}=0$ and when $p=0$ one has:

$$
\boldsymbol{\tau}^{0}(\hat{\boldsymbol{x}}, \boldsymbol{y})=\boldsymbol{G}\left(y_{1}, \boldsymbol{u}^{0}\right)+\frac{\partial u_{i}^{0}}{\partial x_{j}}(0, \hat{\boldsymbol{x}}) \boldsymbol{A} \boldsymbol{\gamma}_{y}\left(\boldsymbol{V}^{i j}\right)(\boldsymbol{y})
$$

where the tensor $\boldsymbol{G}$ is defined by

$$
\boldsymbol{G}\left(y_{1}, \boldsymbol{u}^{0}\right)=\boldsymbol{A}^{I}\left(\boldsymbol{\gamma}_{\boldsymbol{x}}\left(\boldsymbol{u}^{0}(0, \hat{\boldsymbol{x}})\right)+f^{\prime}\left(y_{1}\right) \frac{\partial \boldsymbol{u}^{0}}{\partial x_{1}}(0, \hat{\boldsymbol{x}}) \otimes_{S} \boldsymbol{e}_{1}\right)
$$


* The fields $\boldsymbol{V}^{i j}$ are solution of the following cell problems for $p=0$, resp. for $p \geq 1$ (or for the case of holes since the two situations give rise to the same equations):

i) for $(1,1), 1=1,2,3$ :

$$
\begin{cases}\boldsymbol{d i v}_{y} \boldsymbol{T}^{l 1}=-f^{\prime \prime}\left(y_{1}\right) A_{i 1 l 1} \boldsymbol{e}_{i} & \text { in } Y(\text { resp. in } Y \backslash I) \\ \boldsymbol{T}^{l 1}=\boldsymbol{A} \boldsymbol{\gamma}_{y}\left(\boldsymbol{V}^{l 1}\right) & \text { in } Y(\text { resp. in } Y \backslash I) \\ \boldsymbol{T}^{l 1}\left(\hat{\boldsymbol{x}}, y_{1}, \frac{1}{2}, y_{3}\right) \boldsymbol{e}_{2}=\boldsymbol{T}^{l 1}\left(\hat{\boldsymbol{x}}, y_{1},-\frac{1}{2}, y_{3}\right) \boldsymbol{e}_{2} & \text { on } \mathbb{R} \times \partial \hat{Y} \\ \boldsymbol{T}^{l 1}\left(\hat{\boldsymbol{x}}, y_{1}, y_{2}, \frac{1}{2}\right) \boldsymbol{e}_{3}=\boldsymbol{T}^{l 1}\left(\hat{\boldsymbol{x}}, y_{1}, y_{2},-\frac{1}{2}\right) \boldsymbol{e}_{3} & \text { on } \mathbb{R} \times \partial \hat{Y} \\ \boldsymbol{V}^{l 1}\left(\hat{\boldsymbol{x}}, y_{1}, \frac{1}{2}, y_{3}\right)=\boldsymbol{V}^{l 1}\left(\hat{\boldsymbol{x}}, y_{1},-\frac{1}{2}, y_{3}\right) & \text { on } \mathbb{R} \times \partial \hat{Y} \\ \boldsymbol{V}^{l 1}\left(\hat{\boldsymbol{x}}, y_{1}, y_{2}, \frac{1}{2}\right)=\boldsymbol{V}^{l 1}\left(\hat{\boldsymbol{x}}, y_{1}, y_{2},-\frac{1}{2}\right) & \text { on } \mathbb{R} \times \partial \hat{Y} \\ \lim _{y_{1} \rightarrow \pm \infty} \boldsymbol{T}^{l 1}\left(y_{1}, \hat{\boldsymbol{y}}\right) \boldsymbol{e}_{1}=\mathbf{0} & \text { for } \hat{\boldsymbol{y}} \in \hat{Y}\end{cases}
$$

with the transmission or boundary conditions on $\partial I$ :

\begin{tabular}{|c|c|}
\hline elastic inclusions $(p=0)$ & $\begin{array}{c}\text { elastic inclusion }(p>0) \\
\text { holes }\end{array}$ \\
\hline$\left[\boldsymbol{V}^{l 1}\right]=\left[\boldsymbol{T}^{l 1} \boldsymbol{n}\right]=\mathbf{0}$ & $\boldsymbol{T}^{l 1} \boldsymbol{n}=\mathbf{0}$ \\
\hline
\end{tabular}

ii) for $(1, \mathrm{k})=(1,2) ;(2,2) ;(3,2) ;(1,3) ;(2,3) ;(3,3)$,

$$
\begin{cases}\boldsymbol{d i v}_{y} \boldsymbol{T}^{l k}=\mathbf{0} & \text { in } Y(\text { resp. in } Y \backslash I) \\ \boldsymbol{T}^{l k}=\boldsymbol{A}_{\gamma_{y}}\left(\boldsymbol{V}^{l k}\right) & \text { in } Y(\text { resp. in } Y \backslash I) \\ \boldsymbol{T}^{l k}\left(\hat{\boldsymbol{x}}, y_{1}, \frac{1}{2}, y_{3}\right) \boldsymbol{e}_{2}=\boldsymbol{T}^{l k}\left(\hat{\boldsymbol{x}}, y_{1},-\frac{1}{2}, y_{3}\right) \boldsymbol{e}_{2} & \text { on } \mathbb{R} \times \partial \hat{Y} \\ \boldsymbol{T}^{l k}\left(\hat{\boldsymbol{x}}, y_{1}, y_{2}, \frac{1}{2}\right) \boldsymbol{e}_{3}=\boldsymbol{T}^{l k}\left(\hat{\boldsymbol{x}}, y_{1}, y_{2},-\frac{1}{2}\right) \boldsymbol{e}_{3} & \text { on } \mathbb{R} \times \partial \hat{Y} \\ \boldsymbol{V}^{l k}\left(\hat{\boldsymbol{x}}, y_{1}, \frac{1}{2}, y_{3}\right)=\boldsymbol{V}^{l k}\left(\hat{\boldsymbol{x}}, y_{1},-\frac{1}{2}, y_{3}\right) & \text { on } \mathbb{R} \times \partial \hat{Y} \\ \boldsymbol{V}^{l k}\left(\hat{\boldsymbol{x}}, y_{1}, y_{2}, \frac{1}{2}\right)=\boldsymbol{V}^{l k}\left(\hat{\boldsymbol{x}}, y_{1}, y_{2},-\frac{1}{2}\right) & \text { on } \mathbb{R} \times \partial \hat{Y} \\ \lim _{y_{1} \rightarrow \pm \infty} \boldsymbol{T}^{l k}\left(y_{1}, \hat{\boldsymbol{y}}\right) \boldsymbol{e}_{1}=\mathbf{0} & \text { for } \hat{\boldsymbol{y}} \in \hat{Y}\end{cases}
$$


with the transmission or boundary conditions on $\partial I$ :

\begin{tabular}{|c|c|}
\hline elastic inclusions $(p=0)$ & $\begin{array}{c}\text { elastic inclusion }(p>0) \\
\text { holes }\end{array}$ \\
\hline$\left[\boldsymbol{V}^{l k}\right]=\mathbf{0}$ & $\boldsymbol{T}^{l k} \boldsymbol{n}=-\left(A_{i j l k} n_{j}\right) \boldsymbol{e}_{i}$ \\
{$\left[\boldsymbol{T}^{l k} \boldsymbol{n}\right]=\left(\left(\boldsymbol{A}^{I}-\boldsymbol{A}\right)\left(\boldsymbol{e}_{l} \otimes_{S} \boldsymbol{e}_{k}\right)\right) \boldsymbol{n}$} & \\
\hline
\end{tabular}

Remark 4: For the problems (24) and (26), the following asymptotic behaviours hold [1]:

$$
\lim _{y_{1} \rightarrow \pm \infty} \boldsymbol{V}^{i j}\left(y_{1}, \hat{\boldsymbol{y}}\right)=\boldsymbol{V}^{i j \pm}
$$

Furthermore, the solutions of the cell problems are only defined up to a translation. The uniqueness of $\boldsymbol{V}^{\boldsymbol{i j}}$ can be recovered by adding the following condition:

$$
\boldsymbol{V}^{i j+}+\boldsymbol{V}^{i j-}=\mathbf{0}
$$

Remark 5: As for holes, the concentration of the stresses near the heterogeneities is given by $\boldsymbol{\tau}^{0}$. The inner term $\boldsymbol{\tau}^{0}$ is completely determined by the computation of the cell problems while (18) implies that $\boldsymbol{v}^{1}$ requires the calculation of the constant $\check{\boldsymbol{v}}$. This constant will be determined once $\boldsymbol{u}^{1}$ is known by the following relation:

$$
\check{\boldsymbol{v}}(\hat{\boldsymbol{x}})=\frac{1}{2}\left(\boldsymbol{u}^{1}\left(0^{+}, \hat{\boldsymbol{x}}\right)+\boldsymbol{u}^{1}\left(0^{-}, \hat{\boldsymbol{x}}\right)\right)
$$

Let us also notice that in the case $p=1(24),(26)$ and (30) allow to compute $\boldsymbol{v}^{1}\left(\hat{\boldsymbol{x}}, y_{1}, \hat{\boldsymbol{y}}\right)$ only in $Y \backslash I$. In order to find the displacement field $\boldsymbol{v}^{1}$ in all $Y$ we remark that in $I$ it is the unique solution of the elasticity problem with Dirichlet boundary conditions:

$$
\begin{cases}\boldsymbol{d i v}_{y} \boldsymbol{A}^{I} \boldsymbol{\gamma}_{y}\left(\boldsymbol{v}^{1}\right)=0 & \text { in } I \\ \boldsymbol{v}^{1}=\frac{\partial u_{l}^{0}}{\partial x_{k}}(0, \hat{\boldsymbol{x}}) \boldsymbol{V}^{l k}(\boldsymbol{y})+\check{\boldsymbol{v}}(\hat{\boldsymbol{x}}) & \text { on } \partial I\end{cases}
$$

- outer approximation:

The outer term $\boldsymbol{u}^{1}$ is the solution of the following boundary value prob- 
lem:

$$
\begin{cases}\boldsymbol{d i v} \boldsymbol{\sigma}^{1}=\mathbf{0} & \text { in } \Omega_{0} \backslash \omega \\ \boldsymbol{\sigma}^{1}=\boldsymbol{A} \boldsymbol{\gamma}\left(\boldsymbol{u}^{1}\right) & \text { in } \Omega_{0} \backslash \omega \\ \boldsymbol{\sigma}^{1} \boldsymbol{n}=\mathbf{0} & \text { on } \Gamma_{F} \\ \boldsymbol{u}^{1}=\mathbf{0} & \text { on } \Gamma_{0}\end{cases}
$$

and the transmissions conditions on $\omega$ :

$$
\left\{\begin{array}{l}
{\left[\boldsymbol{u}^{1}\right](\hat{\boldsymbol{x}})=\mathcal{G}_{\boldsymbol{d}}\left(\boldsymbol{u}^{0}(0, \hat{\boldsymbol{x}}) ;\left[\boldsymbol{V}^{i j}\right]^{\infty}\right)} \\
{\left[\boldsymbol{\sigma}^{1} \boldsymbol{e}_{1}\right](\hat{\boldsymbol{x}})=\mathcal{G}_{n S}\left(\boldsymbol{u}^{0}(0, \hat{\boldsymbol{x}}) ; \int_{Y} \boldsymbol{T}^{i j}(\boldsymbol{y}) d \boldsymbol{y}\right)}
\end{array}\right.
$$

where

$$
\left[\boldsymbol{V}^{i j}\right]^{\infty}=\boldsymbol{V}^{i j+}-\boldsymbol{V}^{i j-}
$$

For the different types of inclusions considered, one has:

$$
\mathcal{G}_{\boldsymbol{d}}=\frac{\partial u_{i}^{0}}{\partial x_{j}}(0, \hat{\boldsymbol{x}})\left[\boldsymbol{V}^{i j}\right]^{\infty}
$$

The expression of $\mathcal{G}_{n S}$ depends on the inclusion:

i) in the elastic inclusions case one has:

$$
\mathcal{G}_{n S}=\boldsymbol{d i v}_{x}\left(|I|\left(\boldsymbol{A}-\boldsymbol{A}^{I}\right) \boldsymbol{\gamma}_{x}\left(\boldsymbol{u}^{0}(0, \hat{\boldsymbol{x}})\right)-\frac{\partial u_{i}^{0}}{\partial x_{j}}(0, \hat{\boldsymbol{x}}) \int_{Y} \boldsymbol{T}^{i j}(\boldsymbol{y}) d \boldsymbol{y}\right)
$$

ii) in the holes case one has:

$$
\mathcal{G}_{n S}=\boldsymbol{d i v}_{x}\left(|I| \boldsymbol{A} \boldsymbol{\gamma}_{x}\left(\boldsymbol{u}^{0}(0, \hat{\boldsymbol{x}})\right)-\frac{\partial u_{i}^{0}}{\partial x_{j}}(0, \hat{\boldsymbol{x}}) \int_{Y} \boldsymbol{T}^{i j}(\boldsymbol{y}) d \boldsymbol{y}\right)
$$

Remark 6: In practice, an efficient way to implement the jump conditions in problem (32) is to solve this problem by a domain decomposition type algorithm. The actual procedure will be detailed in section 3 . 


\subsection{Definition of the outer and inner approximations of order $m$}

Once determined the different terms $\mathbf{u}^{i}$ and $\mathbf{v}^{i}$ one can define an outer and an inner approximation of order $m$ :

$$
\begin{aligned}
\mathbf{u}_{m}^{\text {out }} & :=\sum_{i=0}^{i=m} \varepsilon^{i} \mathbf{u}^{i} \\
\mathbf{u}_{m}^{i n n} & :=\sum_{i=0}^{i=m} \varepsilon^{i} \mathbf{v}^{i}
\end{aligned}
$$

The quality of these approximations increases with $m$; here we only consider the cases $m=0$ and $m=1$.

\section{Solution of the first order problem by domain decomposition}

The first order problem (32) will be solved by a domain decomposition type algorithm. In order to describe this method we first recall (see e.g. [16]) the starting point for solving a standard linear elasticity problem by domain decomposition and then we adapt the solution procedure to the problem (32).

\subsection{A linear elasticity model problem}

As it is well known (see e.g. [5]), under the usual regularity, symmetry and coercivity assumptions on $\boldsymbol{A}$, the following linear elasticity problem has a unique solution $\boldsymbol{u} \in \boldsymbol{H}^{1}(\Omega)$ when $\mathbf{F}$ and $\mathbf{u}^{d}$ are regular enough (i.e : $\mathbf{f}^{\Omega} \in \boldsymbol{L}^{2}(\Omega), \mathbf{F} \in \boldsymbol{L}^{2}\left(\Gamma_{0}\right)$ and $\left.\mathbf{u}^{d} \in \boldsymbol{H}^{1 / 2}\left(\Gamma_{0}\right)\right)$

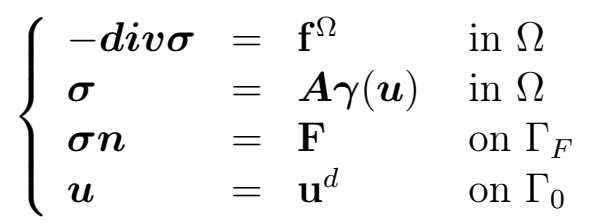

Let the domain $\Omega$ be decomposed in two non overlapping subdomains $\Omega_{1}$ and $\Omega_{2}$ and let $\omega$ be the interface between the subdomains. The objective of the domain decomposition method is to replace the problem on the whole domain by a problem formulated on the interface. The two following conditions hold on the interface:

- continuity of the solution $\boldsymbol{u}$ 
- continuity of the normal stress

We will impose one condition and verify the second. In the following to underline the linear dependence on $\boldsymbol{u}$ we set $\boldsymbol{\sigma}(\boldsymbol{u}):=\boldsymbol{A} \boldsymbol{\gamma}(\boldsymbol{u})$. Let's define $\boldsymbol{\lambda}=\operatorname{Tr}(\boldsymbol{u})$ on the interface $\omega$ where $\boldsymbol{u}$ denotes the solution of problem (38). Solve the problem (38) is equivalent to solve, on each subdomain, the following problems

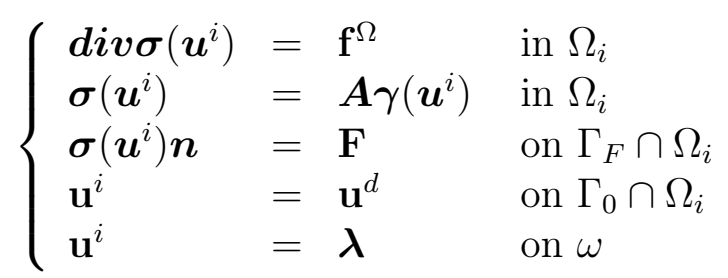

where $\boldsymbol{\lambda}$ is an additional unknown that must be determined in such a way that the normal stresses be continuous on the interface $\omega$, i. e. since $\boldsymbol{n}^{1}=-\boldsymbol{n}^{2}$ :

$$
\boldsymbol{\sigma}\left(\boldsymbol{u}^{1}\right) \boldsymbol{n}^{1}+\boldsymbol{\sigma}\left(\boldsymbol{u}^{2}\right) \boldsymbol{n}^{2}=0
$$

On each subdomain $\Omega_{i}$ we define the Steklov-Poincaré operator $S_{i}$ as follows: for $\boldsymbol{\lambda}$ given $\mathbf{u}_{\lambda}^{i}$ is the solution of

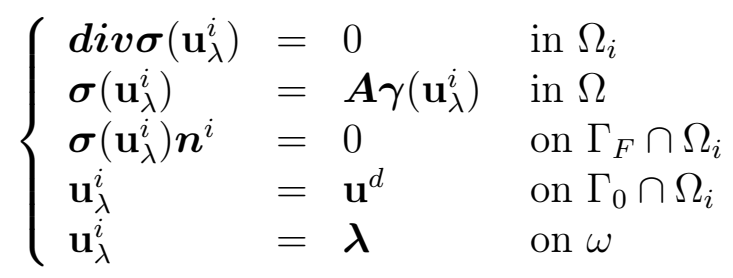

and $S_{i}$ is defined by:

$$
S_{i} \boldsymbol{\lambda}=\boldsymbol{\sigma}\left(\mathbf{u}_{\lambda}^{i}\right) \boldsymbol{n}^{i}
$$

where $\boldsymbol{n}^{i}$ denotes the outer normal to $\Omega_{i}$ on $\omega$.

Notice that using the linearity of (39) one has $\boldsymbol{u}^{i}=\boldsymbol{u}_{0}^{i}+\boldsymbol{u}_{\lambda}^{i}$ where $\boldsymbol{u}_{0}^{i}$ is solution of (39) with $\boldsymbol{u}_{0}^{i}=0$ on $\omega$. The continuity of the normal stress (40) gives

$$
\boldsymbol{\sigma}\left(\mathbf{u}_{\lambda}^{1}\right) \boldsymbol{n}^{1}+\boldsymbol{\sigma}\left(\boldsymbol{u}_{0}^{1}\right) \boldsymbol{n}^{1}+\boldsymbol{\sigma}\left(\mathbf{u}_{\lambda}^{2}\right) \boldsymbol{n}^{2}+\boldsymbol{\sigma}\left(\boldsymbol{u}_{0}^{2}\right) \boldsymbol{n}^{2}=0 .
$$

After introducing the Steklov-Poincare operators we have

$$
S_{1} \boldsymbol{\lambda}+S_{2} \boldsymbol{\lambda}=-\boldsymbol{\sigma}\left(\mathbf{u}_{0}^{1}\right) \boldsymbol{n}^{1}-\boldsymbol{\sigma}\left(\mathbf{u}_{0}^{2}\right) \boldsymbol{n}^{2}
$$

and finally, setting $S=S_{1}+S_{2}$ the system on the interface becomes 


$$
S \boldsymbol{\lambda}=-\boldsymbol{\sigma}\left(\mathbf{u}_{0}^{1}\right) \boldsymbol{n}^{1}-\boldsymbol{\sigma}\left(\mathbf{u}_{0}^{2}\right) \boldsymbol{n}^{2}
$$

Remark 1: The operators $S_{i}$ are linear and continuous from $\boldsymbol{V}(\omega)$ into its dual $\boldsymbol{V}^{\prime}(\omega)$. The definition of these spaces depends on the geometry of $\omega$. When $\omega$ is a closed Lipschitz surface with no intersection with $\partial \Omega$ then $\boldsymbol{V}(\omega)=\boldsymbol{H}^{1 / 2}(\omega)$. Since $\boldsymbol{H}^{1 / 2}(\omega) \subset \boldsymbol{L}^{2}(\omega):=\boldsymbol{H}$ with continuous and dense embedding one can take $\boldsymbol{H} \simeq \boldsymbol{H}^{\prime}$ as pivot space and then $\boldsymbol{V}^{\prime}(\omega)=\boldsymbol{H}^{-1 / 2}(\omega)$. When $\omega \cap \partial \Omega \neq \varnothing$ then an analogous construction is also possible but presents some technical difficulties: essentially it is necessary to use the space denoted by $\boldsymbol{H}_{00}^{1 / 2}(\omega)$ in [17].

Remark 2: $S$ is a symmetric positive defined operator which, after discretization, gives the Schur complement. The Schur complement matrix is not known directly, is rather difficult to construct and is not a sparse matrix, but the product $S \boldsymbol{\lambda}$ is easy to compute as it requires the solution of one problem by subdomain. It is thus convenient to solve the linear system by an iterative method as conjugate gradient or GMRES. GMRES is preferred for its robustness. In addition we will note that even when CG is used a re-orthogonalisation is mandatory for such problems [18].

Remark 3: What was described in this section is only the starting point for a primal domain decomposition method. Several choices of preconditioners are available and the efficiency of the algorithm is indeed strongly depending on this choice. There is a vast literature on the subject, see e.g. [16]. We will use the balancing Neumann-Neumann algorithm [19] which was intensively studied and proved to be robust, [16], [20] for elasticity problems. An alternative is to use a dual method of FETI type [21].

\subsection{Solution of the first order problem}

The script 1 which indicates the first order problem is omitted hereafter. The generic form of the first order problem (32) is given by:

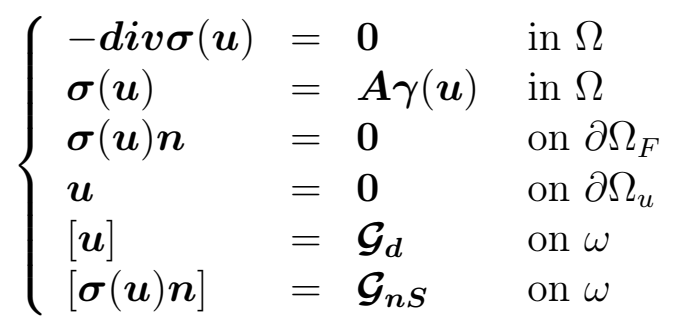


where $\mathcal{G}_{\boldsymbol{d}}$ and $\mathcal{G}_{\boldsymbol{n} \boldsymbol{S}}$ denote the gap in displacements, respectively normal stresses on $\omega$. These data are known and can be computed once the zero order problem is solved. It should be noted that the solution $\boldsymbol{u}$ of (43) does not belong to $\boldsymbol{H}^{1}(\Omega)$. More precisely, let be $\Omega_{1}:=\Omega^{+}, \Omega_{2}:=\Omega^{-}$and :

$$
\boldsymbol{Z}_{0}:=\left\{\boldsymbol{z} \in \boldsymbol{L}^{2}(\Omega) ; \boldsymbol{d i v} \boldsymbol{A} \boldsymbol{\gamma}\left(\left.\boldsymbol{z}\right|_{\Omega_{i}}\right)=0 ;\right\}
$$

then $\boldsymbol{u} \in \boldsymbol{Z}_{0}$. Hence the transmission conditions $[\boldsymbol{u}]=\mathcal{G}_{\boldsymbol{d}}$ and $[\boldsymbol{\sigma}(\boldsymbol{u}) \boldsymbol{n}]=$ $\mathcal{G}_{n S}$ on $\omega$ have to be taken in a weak sense adapting the methods of LionsMagenes [17]. In the Appendix we briefly recall how this can be done.

The actual computation of $\mathcal{G}_{\boldsymbol{d}}$ and $\mathcal{G}_{\boldsymbol{n} \boldsymbol{S}}$ will be described later on, see 3.3; the objective here is to transform the problem in order to obtain a domain decomposition as in (39).

The scripts in the following formulae indicates the subdomain number. Let $\boldsymbol{z}^{i}$ be the solution of the following problems:

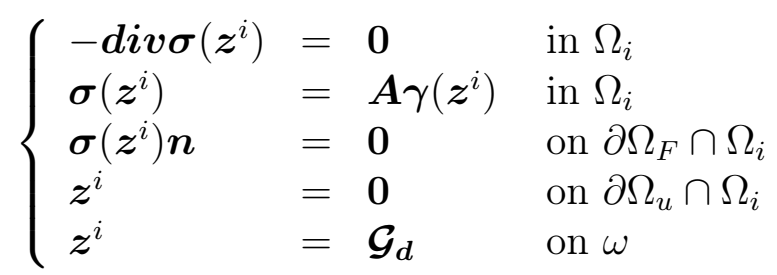

Note that these are two independent problems. Thereafter, on each subdomain, we will look for a solution $\boldsymbol{u}^{i}$ which takes the following form:

$$
\boldsymbol{u}^{i}=\boldsymbol{w}^{i}+\beta_{i} \boldsymbol{z}^{i}
$$

with $\beta_{i}$ two real numbers conveniently chosen. This can be done because the considered operator is linear. With these notations the new unknown of the problem is $\boldsymbol{w}^{i}$. Notice that $-\boldsymbol{d i v} \boldsymbol{\sigma}\left(\boldsymbol{w}^{i}\right)=0$ and that the transmission conditions on $\omega$ for $\boldsymbol{w}^{i}$ are given by:

$$
\left\{\begin{array}{l}
{[\boldsymbol{w}]=[\boldsymbol{u}]-\beta_{1} \mathcal{G}_{\boldsymbol{d}}+\beta_{2} \mathcal{G}_{\boldsymbol{d}}=\left(1-\beta_{1}+\beta_{2}\right) \mathcal{G}_{\boldsymbol{d}}} \\
{[\boldsymbol{\sigma}(\boldsymbol{w}) \boldsymbol{n}]=[\boldsymbol{\sigma}(\boldsymbol{u}) \boldsymbol{n}]+\beta_{1} \boldsymbol{\sigma}\left(\boldsymbol{z}^{1}\right) \boldsymbol{n}-\beta_{2} \boldsymbol{\sigma}\left(\boldsymbol{z}^{2}\right) \boldsymbol{n}=\mathcal{G}_{\boldsymbol{n} \boldsymbol{S}}+\beta_{1} \boldsymbol{\sigma}\left(\boldsymbol{z}^{1}\right) \boldsymbol{n}-\beta_{2} \boldsymbol{\sigma}\left(\boldsymbol{z}^{2}\right) \boldsymbol{n}}
\end{array}\right.
$$

If we choose $1-\beta_{1}+\beta_{2}=0$ then $\boldsymbol{w}$ is continuous on the interface $\omega$ but the normal stress is not and it is the unique non-homogeneous data. As in section 3.1 using the Steklov-Poincaré operator the problem to solve on the interface is

$$
S \boldsymbol{\lambda}=\mathcal{G}_{n \boldsymbol{S}}+\beta_{1} \boldsymbol{\sigma}\left(\boldsymbol{z}^{1}\right) \boldsymbol{n}^{1}-\beta_{2} \boldsymbol{\sigma}\left(\boldsymbol{z}^{2}\right) \boldsymbol{n}^{2}
$$


which can also be written as:

$$
S \lambda=-\boldsymbol{\sigma}\left(\boldsymbol{w}_{0}^{1}\right) \boldsymbol{n}^{1}-\boldsymbol{\sigma}\left(\boldsymbol{w}_{0}^{2}\right) \boldsymbol{n}^{2}+\mathcal{G}_{\boldsymbol{n} \boldsymbol{S}}+\beta_{1} S_{1} \mathcal{G}_{\boldsymbol{d}}-\beta_{2} S_{2} \mathcal{G}_{\boldsymbol{d}}
$$

Notice that equations (42) and (47) differ only on the right hand side. Thus as the operator does not change, the same algorithms may be used to solve the problem with the same performance and no additional analysis is required to prove efficiency. In other words, as for the standard balancing Neumann-Neumann preconditioner $(\mathrm{M})$ the numerical scalability is quasioptimal as the condition number cond $\left(M^{-1} S\right)$ is bounded by a quasi-optimal bound

$$
\operatorname{cond}\left(M^{-1} S\right) \leq \frac{C}{\max _{i}^{2}}\left[1+\log \max \frac{H_{i}}{h_{i}}\right]^{2}
$$

where the constant $C$ is independent of the subdomains diameters $H_{i}$, discretization steps $h_{i}$, aspect ratios $\alpha_{i}$ and elasticity coefficients [19], [16], [20].

\subsection{Implementation issues}

In the framework of domain decomposition algorithms, the implementation of the problem (43) is straightforward as it only requires the solution of an additional problem by subdomain. This problem is of the same type as the standard ones solved by the algorithm.

If we come back to the original equation into consideration (32) the only remaining problem is to compute the input data for the domain decomposition algorithm, that is the right hand sides for the jumps.

$$
\left\{\begin{aligned}
\mathcal{G}_{\boldsymbol{d}} & =\mathcal{G}_{\boldsymbol{d}}\left(\frac{\partial u_{i}}{\partial x_{j}}(0, \hat{\boldsymbol{x}}) ;\left[\boldsymbol{V}^{i j}\right]^{\infty}\right) \\
\mathcal{G}_{\boldsymbol{n} \boldsymbol{S}} & =\mathcal{G}_{\boldsymbol{n} \boldsymbol{S}}\left(\frac{\partial^{2} u_{i}}{\partial x_{\alpha} \partial x_{j}}(0, \hat{\boldsymbol{x}}) ; \int_{Y} \boldsymbol{T}^{i j}(\boldsymbol{y}) d \boldsymbol{y}\right)
\end{aligned}\right.
$$

From a mathematical point of view these quantities are data as they depend on the solution of the zero order problem. From a computational point of view this task is rather delicate. Indeed the solution of the zero order problem was obtained by a standard finite element method. So the solution $\boldsymbol{u}^{0}$ is $\mathcal{C}^{0}$ that means no continuity of the derivatives. The actual computations require first order derivatives for stresses that means second order derivatives for displacements. As it is well known, see e.g [22] Chap. 4, Sec 4.2 , the calculation of derivatives numerically is a hazardous operation 
since it is inherently sensitive, as small perturbations in data can cause large changes in result (in other words numerical differentiation is ill-conditioned). For this reason, to approximate derivative of a function whose values are known only at discrete set of points, a good approach is to fit some smooth function to given data and then differentiate the approximating function. If the given data are sufficiently smooth, then interpolation may be appropriate; here $\boldsymbol{u}^{0} \in \mathcal{C}^{0}$ and hence it is worth regularizing before computing derivatives. Let us mention, with [22], Chap 6, Sect 6.7 that the choice of the better approximation "is in the realm of the art rather than in the science of numerical analysis. Intuition - meaning experience, really - and general physical knowledge of the problem must be used."

\section{- computation of $\mathcal{G}_{d}$}

In practice the value of the displacement derivative at a node is a weighted average of the values of the derivatives corresponding to the different elements containing the node. The weight considered is the ratio between the surface of the actual element and the surface of all elements containing the node.

\section{- computation of $\mathcal{G}_{n S}$}

Let's first notice that, for the sake of simplicity, all equations appear as partial differential equations, instead of in variational form. What is needed in practice, once the variational form is introduced, is $\int_{\Gamma} \mathcal{G}_{n \boldsymbol{S}} v d v$. As the integral is computed element by element at a first glance it is not mandatory to regularize before integrating. Nevertheless our choice was to use the same regularization as for the computation of $\mathcal{G}_{\boldsymbol{d}}$.

Remark 4: The algorithm described in this section was specially designed to handle jumps in displacements and normal stresses on the internal surface $\omega$. In addition, as mentioned previously, it has the ability to consider a large number of subdomains. This can be illustrated by the following results obtained by solving the first order problem (see section 4) on a mesh with mesh-size $h=1 / 120$ (57600 P2-Lagrange elements , 231602 degrees of freedom). 


\begin{tabular}{|c|c|}
\hline Number of subdomains & Number of iterations \\
\hline 6 & 24 \\
10 & 25 \\
48 & 29 \\
64 & 26 \\
\hline
\end{tabular}

\section{Validation}

The objective of the validation is to study, in an appropriate norm, the error between the exact solution and the solution obtained by asymptotic expansions (zero order and first order). This error depends both on $\boldsymbol{u}_{h}^{\varepsilon}$ and $h$ the discretisation step. Our interest is to focus on the dependence of the error on $\varepsilon$ and $h$. So for all the computations the approximation error (which depends on $h$ ) has to be negligible, in other words $h$ has to be much smaller than $\varepsilon$. Hence there are several difficulties in the validation process:

i) The domain depends on the value of $\varepsilon$. For the test problem and for decreasing values of $\varepsilon$ the number of heterogeneities increases, thus the domain changes and it is impossible to obtain a uniform refinement of the mesh with respect to $\varepsilon$.

ii) The analytical solution of the original test problem is not known. We use instead a reference solution $\boldsymbol{u}_{h}^{\varepsilon}$ obtained with standard $P 2$ finite elements on a suitable mesh $\Omega_{h}^{\varepsilon}$, fine enough so that the error between the exact solution and the computed one is negligible in comparison with the error due to the use of asymptotic expansions. Let us recall that when the solution $\boldsymbol{u}^{\varepsilon}$ has enough regularity the $H^{1}$ norm is used to measure the error and one has, for a fixed $\varepsilon$ and for a regular mesh with discretization step $h:\left\|\boldsymbol{u}^{\varepsilon}-\boldsymbol{u}_{h}^{\varepsilon}\right\|_{H^{1}\left(\Omega^{\varepsilon}\right)} \leq C h^{2}$. The solution of our target problem is not regular enough, this is why an $L^{2}$ norm will be preferred as detailed here in Sect. 4.3

iii) The definition of $\Omega^{i n, \varepsilon}$ depends on $\eta(\varepsilon)$. The function $\eta(\varepsilon)$ is not a given datum, it must only verify (3) and, from a theoretical point, it has a crucial importance. 
iv) The boundary of the domain $\Omega$ has corners: the numerical solution of the different problems has boundary layers whose effects can perturb the quality of the results.

The validation is achieved through the following steps:

1. Description of the test problem

2. Numerical solution of the cell problem

3. Validation at the macroscopic scale

4. Validation at the microscopic scale

\subsection{Description of the test problem}

Let $\Omega=(-L, L) \times(-H, H)$ be a plane domain containing $\mathcal{N}(\varepsilon)=2 H \varepsilon^{-1}$ identical discs of diameter $\varepsilon d$, arranged periodically (with period $\varepsilon$ ), along the line $\omega$ of equation $x_{1}=0$. Outside the heterogeneities the structure is constituted of an elastic homogeneous isotropic material, characterized by its Young modulus $E$ and Poisson coefficient $\nu$. The boundary $\Gamma_{0}$ of the structure is clamped and a density force $\boldsymbol{F}$ is applied on the complementary part $\Gamma_{F}$. There are no body forces (see Fig. 6). We will consider a $2 \mathrm{D}$ plane stress problem. The heterogeneities can be holes or elastic inclusions; in this

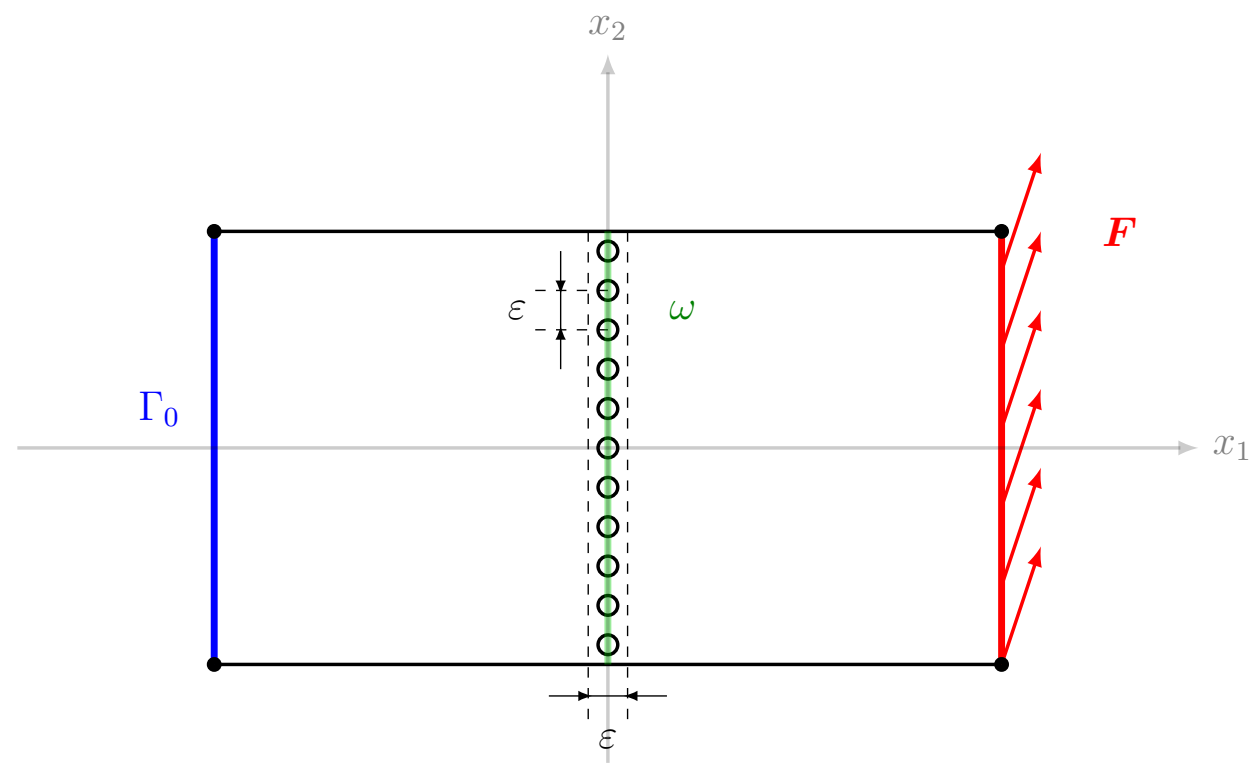

Figure 6: Test Problem 
case the elastic homogeneous isotropic material forming the heterogeneities is characterized by the Young modulus $E^{\varepsilon, I}=\varepsilon^{p} E^{I}$ and the Poisson coefficient $\nu^{\varepsilon, I}=\nu$. In all cases the problem is solved by a standard $P 2$ finite element method and we take $L=1$ and $H=0.5$.

\subsection{Numerical solution of the cell problems}

The transmission conditions of the first-order outer problem (32) depend on the function $f\left(y_{1}\right)$ and on the solution of the cell problems posed on the unbounded domain $Y$. In practice we will use a bounded domain $Y_{L^{\infty}}=$ ] $\frac{-L^{\infty}}{2}, \frac{L^{\infty}}{2}[\times] \frac{-1}{2}, \frac{1}{2}$ [ with a $L^{\infty}>0$ big enough. The domain $Y_{L^{\infty}}$ contains a circular heterogeneity of diameter $d$ and centred at the origin. At first we numerically study, in the case of elastic inclusions with $p=0$ and in the case of holes (or of elastic inclusions with $p>1$ ), the robustness of the coefficients $\left[\boldsymbol{V}^{i j}\right]$ and $\int_{Y} \boldsymbol{T}^{i j} d \boldsymbol{y}$ with respect to:

i) the size $L^{\infty}$ needed to approximate $Y$ for the computation of $\boldsymbol{V}^{i j}$,

ii) the choice of the function $f\left(y_{1}\right)$,

iii) the choice of the diameter $d$,

iv) In the case of elastic inclusions with $p=0$ the dependence on $E^{I} / E$.

The cell problems (24)-(27) are solved by a standard P2 finite elements method.

\subsubsection{Dependence on $L^{\infty}$}

The cell problems are solved by gradually increasing the length value $L^{\infty}$. As odd function of class $\mathcal{C}^{2}(\mathbb{R})$ satisfying $(19)$ a polynomial of order 5 for $a \leq\left|y_{1}\right| \leq b$ is choosed. The results are obtained with $d=0.60, a=1.5$, $b=3 a, E=1, \nu=0.25$ (and also with $E^{I}=0.5, \nu^{I}=0.25$ in the case $p=0)$. They show that for $L^{\infty} \geq 2.01 b$ the coefficients $\left[\boldsymbol{V}^{i j}\right]$ and $\int_{Y} \boldsymbol{T}^{i j} d \boldsymbol{y}$ are stable (see Tables 1, 2, 3 and 4 below where only non-zero coefficients are given). 


\begin{tabular}{|c|c|c|c|c|}
\hline$L^{\infty}$ & {$\left[V_{1}^{11}\right]^{\infty}$} & {$\left[V_{2}^{12}\right]^{\infty}$} & {$\left[V_{2}^{21}\right]^{\infty}$} & {$\left[V_{1}^{22}\right]^{\infty}$} \\
\hline $2 \mathrm{~b}+0.001 \mathrm{~b}$ & 0.2211195 & 0.2420530 & 0.2420514 & 0.0562849 \\
$2 \mathrm{~b}+0.005 \mathrm{~b}$ & 0.2211221 & 0.2420530 & 0.2420524 & 0.0562849 \\
$2 \mathrm{~b}+0.010 \mathrm{~b}$ & 0.2211242 & 0.2420530 & 0.2420527 & 0.0562849 \\
$2 \mathrm{~b}+0.020 \mathrm{~b}$ & 0.2211258 & 0.2420530 & 0.2420527 & 0.0562849 \\
$2 \mathrm{~b}+0.030 \mathrm{~b}$ & 0.2211261 & 0.2420530 & 0.2420527 & 0.0562849 \\
$2 \mathrm{~b}+0.040 \mathrm{~b}$ & 0.2211262 & 0.2420530 & 0.2420527 & 0.0562849 \\
$2 \mathrm{~b}+0.050 \mathrm{~b}$ & 0.2211262 & 0.2420530 & 0.2420527 & 0.0562849 \\
$2 \mathrm{~b}+0.060 \mathrm{~b}$ & 0.2211262 & 0.2420530 & 0.2420527 & 0.0562849 \\
$2 \mathrm{~b}+0.070 \mathrm{~b}$ & 0.2211262 & 0.2420530 & 0.2420527 & 0.0562849 \\
$2 \mathrm{~b}+0.080 \mathrm{~b}$ & 0.2211262 & 0.2420530 & 0.2420527 & 0.0562849 \\
$2 \mathrm{~b}+0.090 \mathrm{~b}$ & 0.2211262 & 0.2420530 & 0.2420527 & 0.0562849 \\
$2 \mathrm{~b}+0.100 \mathrm{~b}$ & 0.2211262 & 0.2420530 & 0.2420527 & 0.0562849 \\
\hline
\end{tabular}

Table 1: Variation of $\left[V_{k}^{i j}\right]^{\infty}$ with $L^{\infty}$ : elastic inclusions case

\begin{tabular}{|c|c|c|c|c|}
\hline$L^{\infty}$ & $\int_{Y} T_{22}^{11} d \boldsymbol{y}$ & $\int_{Y} T_{12}^{12} d \boldsymbol{y}$ & $\int_{Y} T_{11}^{22} d \boldsymbol{y}$ & $\int_{Y} T_{22}^{22} d \boldsymbol{y}$ \\
\hline $2 \mathrm{~b}+0.001 \mathrm{~b}$ & -0.0010703 & 0.0561855 & 0.0374570 & -0.0296238 \\
$2 \mathrm{~b}+0.005 \mathrm{~b}$ & -0.0010703 & 0.0561855 & 0.0374570 & -0.0296238 \\
$2 \mathrm{~b}+0.010 \mathrm{~b}$ & -0.0010703 & 0.0561855 & 0.0374570 & -0.0296238 \\
$2 \mathrm{~b}+0.050 \mathrm{~b}$ & -0.0010703 & 0.0561855 & 0.0374570 & -0.0296238 \\
$2 \mathrm{~b}+0.100 \mathrm{~b}$ & -0.0010703 & 0.0561855 & 0.0374570 & -0.0296238 \\
\hline
\end{tabular}

Table 2: Variation of $\int_{Y} T_{k l}^{i j} d \boldsymbol{y}$ with $L^{\infty}$ : elastic inclusions case

\begin{tabular}{|c|c|c|c|c|}
\hline$L^{\infty}$ & {$\left[V_{1}^{11}\right]^{\infty}$} & {$\left[V_{2}^{12}\right]^{\infty}$} & {$\left[V_{2}^{21}\right]^{\infty}$} & {$\left[V_{1}^{22}\right]^{\infty}$} \\
\hline $2 \mathrm{~b}+0.001 \mathrm{~b}$ & 1.0485810 & 1.7924989 & 1.7924975 & 0.2748645 \\
$2 \mathrm{~b}+0.005 \mathrm{~b}$ & 1.0485836 & 1.7924989 & 1.7924985 & 0.2748645 \\
$2 \mathrm{~b}+0.010 \mathrm{~b}$ & 1.0485857 & 1.7924989 & 1.7924988 & 0.2748645 \\
$2 \mathrm{~b}+0.050 \mathrm{~b}$ & 1.0485877 & 1.7924989 & 1.7924988 & 0.2748645 \\
$2 \mathrm{~b}+0.100 \mathrm{~b}$ & 1.0485877 & 1.7924989 & 1.7924988 & 0.2748645 \\
\hline
\end{tabular}

Table 3: Variation of $\left[V_{k}^{i j}\right]^{\infty}$ with $L^{\infty}$ : holes case

\subsubsection{Dependence on $f$}

We consider two functions $f_{1}\left(y_{1} ; a, b\right)$ et $f_{2}\left(y_{2} ; a, b\right)$ which satisfy the condition (19) (we give their definition only for $y_{1}>0$ ): 


\begin{tabular}{|c|c|c|c|c|}
\hline$L^{\infty}$ & $\int_{Y} T_{22}^{11} d \boldsymbol{y}$ & $\int_{Y} T_{12}^{12} d \boldsymbol{y}$ & $\int_{Y} T_{11}^{22} d \boldsymbol{y}$ & $\int_{Y} T_{22}^{22} d \boldsymbol{y}$ \\
\hline $2 \mathrm{~b}+0.001 \mathrm{~b}$ & -0.0135654 & 0.1130969 & 0.0753980 & -0.2005621 \\
$2 \mathrm{~b}+0.005 \mathrm{~b}$ & -0.0135654 & 0.1130969 & 0.0753980 & -0.2005621 \\
$2 \mathrm{~b}+0.010 \mathrm{~b}$ & -0.0135654 & 0.1130969 & 0.0753980 & -0.2005621 \\
$2 \mathrm{~b}+0.050 \mathrm{~b}$ & -0.0135654 & 0.1130969 & 0.0753980 & -0.2005621 \\
$2 \mathrm{~b}+0.100 \mathrm{~b}$ & -0.0135654 & 0.1130969 & 0.0753980 & -0.2005621 \\
\hline
\end{tabular}

Table 4: Variation of $\int_{Y} T_{k l}^{i j} d \boldsymbol{y}$ with $L^{\infty}$ : holes case

\begin{tabular}{c|c} 
& for $a \leq y_{1} \leq b$ \\
\hline$f_{1}\left(y_{1} ; a, b\right)$ & $\left(y_{1}-a\right)^{3}\left(A y_{1}^{2}+B y_{1}+C\right)$ \\
$f_{2}\left(y_{1} ; a, b\right)$ & $\left(y_{1}-a\right)^{3}\left(\alpha y_{1}^{3}+\beta y_{1}^{2}+\gamma y_{1}+\delta\right)$
\end{tabular}

The comparison of $\left[\boldsymbol{V}^{i j, f_{1}}\right]$ with $\left[\boldsymbol{V}^{i j, f_{2}}\right]$ and of $\int_{Y} \boldsymbol{T}^{i j, f_{1}} d \boldsymbol{y}$ with $\int_{Y} \boldsymbol{T}^{i j, f_{2}} d \boldsymbol{y}$ confirms the independence of these coefficients from the choice of $f$. The values of the coefficients calculated for both $f_{1}$ and $f_{2}$ are given in the case of elastic inclusions with $p=0$ in Tab.5 (only non-zero coefficients are given) where $a=0.5, b=4 a, E=1, E^{I}=0.5, \nu=\nu^{I}=0.25, d=0.60$ and $L^{\infty}=10$. The analogous situation for the holes is given in Tab.6 where :

\begin{tabular}{|c|c|c|c|c|c|}
\hline \multicolumn{2}{|c|}{$\left[V_{1}^{11}\right]^{\infty}$} & \multicolumn{2}{c|}{$\left[V_{2}^{21}\right]^{\infty}$} & \multicolumn{2}{c|}{$\int_{Y} T_{22}^{11}$} \\
\hline$f_{1}$ & $f_{2}$ & $f_{1}$ & $f_{2}$ & $f_{1}$ & $f_{2}$ \\
\hline 0.2211406 & 0.2211249 & 0.2420602 & 0.2420444 & -0.0010698 & -0.0010739 \\
\hline
\end{tabular}

Table 5: Result of the coefficient calculation: elastic $p=0$ case

$a=0.5, b=4 a, E=1, \nu=0.25, d=0.60$ and $L^{\infty}=10$.

Note that condition (19) implies that the function $f$ is involved in the

\begin{tabular}{|c|c|c|c|c|c|}
\hline \multicolumn{2}{|c|}{$\left[V_{1}^{11}\right]^{\infty}$} & \multicolumn{2}{c|}{$\left[V_{2}^{21}\right]^{\infty}$} & \multicolumn{2}{c|}{$\int_{Y} T_{22}^{11}$} \\
\hline$f_{1}$ & $f_{2}$ & $f_{1}$ & $f_{2}$ & $f_{1}$ & $f_{2}$ \\
\hline 1.0485342 & 1.0485524 & 1.7922510 & 1.7922715 & -0.0135626 & -0.0136194 \\
\hline
\end{tabular}

Table 6: Result of the coefficient calculation: holes case

loading in problems $P^{11}$ and $P^{21}$. For both problems, the influence of the function $f$ on this loading is shown in figures (7), (8), (9) and (10). 


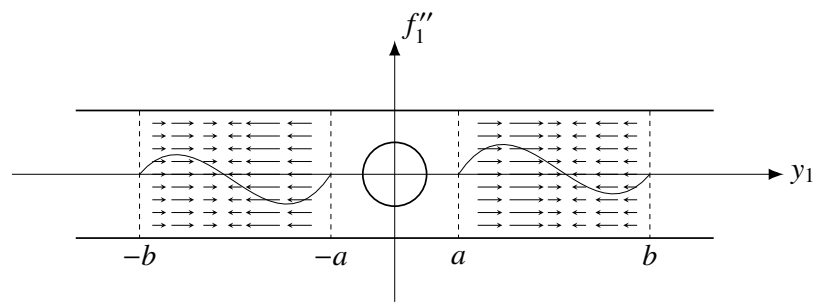

Figure 7: loading for $P^{11}: f=f_{1}$

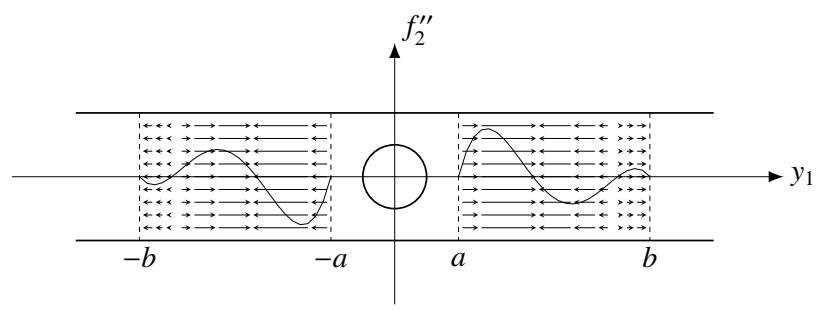

Figure 8: loading for $P^{11}: f=f_{2}$

\subsubsection{Dependence on $d$}

It is also interesting to study the dependence of $\left[\boldsymbol{V}^{i j}\right]^{\infty}$ and of $\int_{Y} \boldsymbol{T}^{i j} d \boldsymbol{y}$ on the diameter $d$. In the case of holes (or equivalently of elastic inclusions with $p>0$ ) the influence can be very important when the diameter becomes almost of the same order as the period or becomes very small as one can see in Tables 7 and 8.

For these comparisons we have chosen $a=1.5, b=3 a, E=1, \nu=0.25$ and $L^{\infty}=10$. The corresponding results obtained for elastic inclusion with $p=0$ when $a=1.5, b=3 a, E=1, \nu=0.25, E^{I}=0.5, \nu^{I}=0.25$ and $L^{\infty}=10$ are given in Table 9 and Table 10 .

\subsubsection{Dependence on $E^{I} / E$ in the case of elastic inclusions with $p=0$}

In the case of elastic inclusions with $p=0$ it is also interesting to study the dependence of $\left[\boldsymbol{V}^{i j}\right]^{\infty}$ and of $\int_{Y} \boldsymbol{T}^{i j} d \boldsymbol{y}$ on $E^{I} / E$. We have considered the case where $\mathrm{a}=1.5, \mathrm{~b}=3^{*} \mathrm{a}, d=0.60, \mathrm{E}=1, \nu=0.25, \nu^{I}=0.25, L^{\infty}=10$. It is interesting to notice the difference of situations when $E^{I} / E$ decreases 


\begin{tabular}{|c|c|c|c|c|}
\hline$d / 2$ & {$\left[V_{1}^{11}\right]^{\infty}$} & {$\left[V_{2}^{12}\right]^{\infty}$} & {$\left[V_{2}^{21}\right]^{\infty}$} & {$\left[V_{1}^{22}\right]^{\infty}$} \\
\hline 0.010 & 0.0008996 & 0.0010023 & 0.0010020 & 0.0001476 \\
0.050 & 0.0226144 & 0.0255398 & 0.0255396 & 0.0038205 \\
0.100 & 0.0918260 & 0.1074091 & 0.1074089 & 0.0169884 \\
0.150 & 0.2124450 & 0.2633347 & 0.2633345 & 0.0439429 \\
0.200 & 0.3949921 & 0.5307941 & 0.5307939 & 0.0905881 \\
0.250 & 0.6601650 & 0.9858120 & 0.9858119 & 0.1641006 \\
0.300 & 1.0486034 & 1.7922971 & 1.7922969 & 0.2748658 \\
0.350 & 1.6469987 & 3.3681096 & 3.3681096 & 0.4433453 \\
0.400 & 2.6773118 & 7.1156680 & 7.1156679 & 0.7231693 \\
0.450 & 5.0071671 & 21.4732286 & 21.4732255 & 1.3301432 \\
0.490 & 14.5258667 & 214.6823144 & 214.6821550 & 3.7303738 \\
\hline
\end{tabular}

Table 7: Dependence on the diameter for $\left[\boldsymbol{V}^{i j}\right]^{\infty}$ : the hole case

\begin{tabular}{|c|c|c|c|c|}
\hline$d / 2$ & $\int_{Y} T_{22}^{11} d \boldsymbol{y}$ & $\int_{Y} T_{12}^{12} d \boldsymbol{y}$ & $\int_{Y} T_{11}^{22} d \boldsymbol{y}$ & $\int_{Y} T_{22}^{22} d \boldsymbol{y}$ \\
\hline 0.010 & 0.0000824 & 0.0001257 & 0.0000838 & -0.0005835 \\
0.050 & 0.0019553 & 0.0031416 & 0.0020944 & -0.0141852 \\
0.100 & 0.0063660 & 0.0125663 & 0.0083775 & -0.0516133 \\
0.150 & 0.0097795 & 0.0282742 & 0.0188495 & -0.1000315 \\
0.200 & 0.0087039 & 0.0502653 & 0.0335102 & -0.1464124 \\
0.250 & 0.0010034 & 0.0785395 & 0.0523597 & -0.1814430 \\
0.300 & -0.0135626 & 0.1130969 & 0.0753980 & -0.2005602 \\
0.350 & -0.0337021 & 0.1539375 & 0.1026250 & -0.2026228 \\
0.400 & -0.0574308 & 0.2010612 & 0.1340408 & -0.1879331 \\
0.450 & -0.0835749 & 0.2544681 & 0.1696454 & -0.1564380 \\
0.490 & -0.1054995 & 0.3017174 & 0.2011450 & -0.1191938 \\
\hline
\end{tabular}

Table 8: Dependence on the diameter for $\int_{Y} \boldsymbol{T}^{i j} d \boldsymbol{y}$ : the hole case 


\begin{tabular}{|c|c|c|c|c|}
\hline$d / 2$ & {$\left[V_{1}^{11}\right]$} & {$\left[V_{2}^{12}\right]$} & {$\left[V_{2}^{21}\right]$} & {$\left[V_{1}^{22}\right]$} \\
\hline 0.010 & 0.0002307 & 0.0002376 & 0.0002374 & 0.0000528 \\
0.050 & 0.0057838 & 0.0059676 & 0.0059673 & 0.0013263 \\
0.100 & 0.0232345 & 0.0241464 & 0.0241462 & 0.0054055 \\
0.150 & 0.0526687 & 0.0553526 & 0.0553523 & 0.0125179 \\
0.200 & 0.0946909 & 0.1009027 & 0.1009024 & 0.0230667 \\
0.250 & 0.1503118 & 0.1625104 & 0.1625101 & 0.0375075 \\
0.300 & 0.2211246 & 0.2420470 & 0.2420467 & 0.0562854 \\
0.350 & 0.3113063 & 0.3430488 & 0.3430488 & 0.0802322 \\
0.400 & 0.4220883 & 0.4629466 & 0.4629466 & 0.1090197 \\
0.450 & 0.5613008 & 0.6011278 & 0.6011279 & 0.1434687 \\
0.490 & 0.7019167 & 0.7212938 & 0.7212938 & 0.1757700 \\
\hline
\end{tabular}

Table 9: Dependence on the diameter for $\left[\boldsymbol{V}^{i j}\right]^{\infty}$ : the elastic inclusion case

\begin{tabular}{|c|c|c|c|c|}
\hline$d / 2$ & $\int_{Y} T_{22}^{11} d \boldsymbol{y}$ & $\int_{Y} T_{12}^{12} d \boldsymbol{y}$ & $\int_{Y} T_{11}^{22} d \boldsymbol{y}$ & $\int_{Y} T_{22}^{22} d \boldsymbol{y}$ \\
\hline 0.010 & 0.0000053 & 0.0000624 & 0.0000416 & -0.0000658 \\
0.050 & 0.0001276 & 0.0015607 & 0.0010405 & -0.0016190 \\
0.100 & 0.0004300 & 0.0062428 & 0.0041619 & -0.0061381 \\
0.150 & 0.0006925 & 0.0140464 & 0.0093643 & -0.0126149 \\
0.200 & 0.0006464 & 0.0249713 & 0.0166476 & -0.0196930 \\
0.250 & 0.0000752 & 0.0390177 & 0.0260118 & -0.0258496 \\
0.300 & -0.0010828 & 0.0564577 & 0.0376385 & -0.0296633 \\
0.350 & -0.0025660 & 0.0768453 & 0.0512302 & -0.0297693 \\
0.400 & -0.0037308 & 0.1003693 & 0.0669129 & -0.0253244 \\
0.450 & -0.0033531 & 0.1270299 & 0.0846866 & -0.0156543 \\
0.490 & -0.0003102 & 0.1506167 & 0.1004111 & -0.0037496 \\
\hline
\end{tabular}

Table 10: Dependence on the diameter of $\int_{Y} \boldsymbol{T}^{i j} d \boldsymbol{y}$ : the elastic inclusion case 


\begin{tabular}{|c|c|c|c|c|}
\hline$E^{I} / E$ & {$\left[V_{1}^{11}\right]$} & {$\left[V_{2}^{12}\right]$} & {$\left[V_{2}^{21}\right]$} & {$\left[V_{1}^{22}\right]$} \\
\hline 0.100 & 0.7365879 & 1.0390793 & 1.0390789 & 0.1915956 \\
0.200 & 0.5399045 & 0.6856242 & 0.6856239 & 0.1395623 \\
0.300 & 0.4019328 & 0.4772223 & 0.4772220 & 0.1033120 \\
0.400 & 0.2997924 & 0.3396756 & 0.3396752 & 0.0766644 \\
0.500 & 0.2211262 & 0.2420530 & 0.2420527 & 0.0562849 \\
0.600 & 0.1586740 & 0.1691533 & 0.1691530 & 0.0402175 \\
0.700 & 0.1078900 & 0.1126290 & 0.1126287 & 0.0272399 \\
0.800 & 0.0657828 & 0.0675133 & 0.0675130 & 0.0165498 \\
0.900 & 0.0303030 & 0.0306650 & 0.0306647 & 0.0075988 \\
1.000 & -0.0000003 & -0.0000000 & -0.0000003 & -0.0000000 \\
2.000 & -0.1624511 & -0.1528339 & -0.1528342 & -0.0397685 \\
3.000 & -0.2285392 & -0.2099850 & -0.2099853 & -0.0552880 \\
4.000 & -0.2643981 & -0.2399011 & -0.2399014 & -0.0634781 \\
5.000 & -0.2869096 & -0.2583075 & -0.2583078 & -0.0685186 \\
6.000 & -0.3023570 & -0.2707759 & -0.2707762 & -0.0719263 \\
7.000 & -0.3136148 & -0.2797811 & -0.2797813 & -0.0743811 \\
8.000 & -0.3221839 & -0.2865902 & -0.2865905 & -0.0762323 \\
9.000 & -0.3289248 & -0.2919194 & -0.2919196 & -0.0776776 \\
10.000 & -0.3343663 & -0.2962038 & -0.2962041 & -0.0788369 \\
\hline
\end{tabular}

Table 11: Dependence on $E^{I} / E$ for $\left[\boldsymbol{V}^{i j}\right]^{\infty}$ 


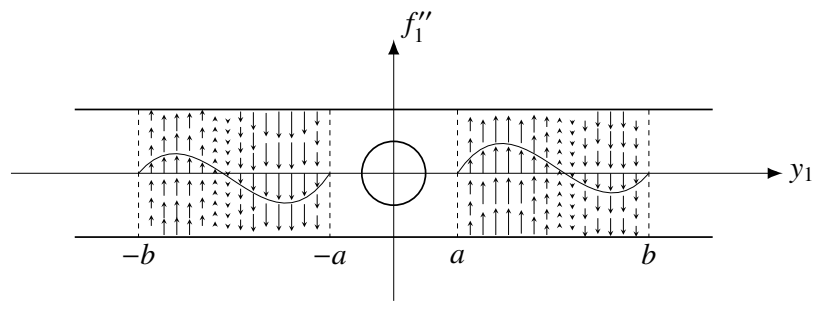

Figure 9: loading for $P^{21}: f=f_{1}$

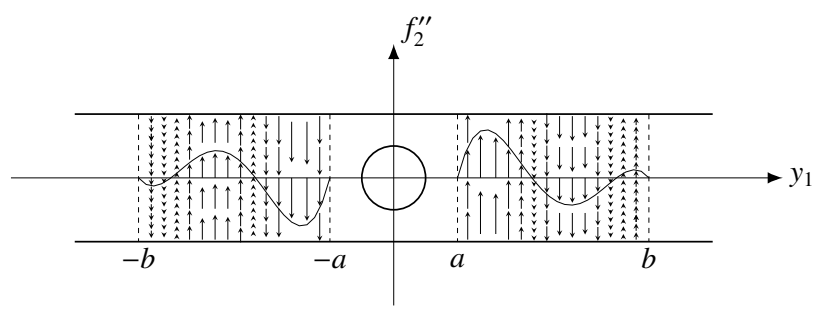

Figure 10: loading for $P^{21}: f=f_{2}$

or increases, see Tables 11,12 . When $E^{I} / E>1$ (i.e. the material of the inclusion is more rigid than the surrounding material) then the coefficients $\left[\boldsymbol{V}^{i j}\right]^{\infty}$ become all negative and, in absolute value, monotonically increasing. In all other situations these coefficients are positive.

\subsection{Validation at the macroscopic scale}

The validation at the macroscopic scale should be performed by comparing $\boldsymbol{u}_{0}^{\text {out }}:=\boldsymbol{u}^{0}$ and $\boldsymbol{u}_{1}^{\text {out }}:=\boldsymbol{u}^{0}+\varepsilon \boldsymbol{u}^{1}$ with $\boldsymbol{u}^{\varepsilon}$; however we can only compute $\boldsymbol{u}_{h}^{\varepsilon}, \boldsymbol{u}_{h}^{0}$ and $\boldsymbol{u}_{h}^{1}$. Since $\boldsymbol{u}^{1}$ does not belong to $\boldsymbol{H}^{1}(\Omega)$ but only to $\boldsymbol{Z}_{0} \subset \boldsymbol{L}^{2}(\Omega)$ (as it has been observed in Sect. 3.2) the relative norm errors $\left\|\boldsymbol{u}_{h}^{\varepsilon}-\boldsymbol{u}_{0, h}^{\text {out }}\right\| /$ $\left\|\boldsymbol{u}_{h}^{\varepsilon}\right\|$ and $\left\|\boldsymbol{u}_{h}^{\varepsilon}-\boldsymbol{u}_{1, h}^{\text {out }}\right\| /\left\|\boldsymbol{u}_{h}^{\varepsilon}\right\|$ are computed using the norm of $\boldsymbol{L}^{2}\left(\Omega^{\varepsilon}\right)$. The numerical reference solution $\boldsymbol{u}_{h}^{\varepsilon}$ is computed on a mesh $\Omega_{h}^{\varepsilon}$ and the numerical asymptotic solutions $\boldsymbol{u}_{0, h}^{\text {out }}:=\boldsymbol{u}_{h}^{0}$ and $\boldsymbol{u}_{1, h}^{\text {out }}:=\boldsymbol{u}_{h}^{0}+\varepsilon \boldsymbol{u}_{h}^{1}$ are computed on a coarse mesh $\Omega_{h}$. Hence in order to compare these numerical approximations one has at first to determine by interpolation the nodal values of $\boldsymbol{u}_{0, h}^{\text {out }}$ and of $\boldsymbol{u}_{1, h}^{\text {out }}$ on $\Omega_{h}^{\varepsilon}$. We have the following obvious estimate for $i=0,1$ :

$$
\left\|\boldsymbol{u}^{\varepsilon}-\boldsymbol{u}_{i}^{\text {out }}\right\| \leq\left\|\boldsymbol{u}^{\varepsilon}-\boldsymbol{u}_{h}^{\varepsilon}\right\|+\left\|\boldsymbol{u}_{h}^{\varepsilon}-\boldsymbol{u}_{i h}^{\text {out }}\right\|+\left\|\boldsymbol{u}_{i h}^{\text {out }}-\boldsymbol{u}_{i}^{\text {out }}\right\|
$$

Since we just compute the term $\left\|\boldsymbol{u}_{h}^{\varepsilon}-\boldsymbol{u}_{i h}^{\text {out }}\right\|$ we should choose a discretization step $h$ so that the approximation errors $\left\|\boldsymbol{u}^{\varepsilon}-\boldsymbol{u}_{h}^{\varepsilon}\right\|$ and $\left\|\boldsymbol{u}_{i}^{\text {out }}-\boldsymbol{u}_{i}^{\text {out }}\right\|$ be 


\begin{tabular}{|c|c|c|c|c|}
\hline$E^{I} / E$ & $\int_{Y} T_{22}^{11} d \boldsymbol{y}$ & $\int_{Y} T_{12}^{12} d \boldsymbol{y}$ & $\int_{Y} T_{11}^{22} d \boldsymbol{y}$ & $\int_{Y} T_{22}^{22} d \boldsymbol{y}$ \\
\hline 0.100 & -0.0079452 & 0.1011340 & 0.0674226 & -0.1470031 \\
0.200 & -0.0048919 & 0.0898969 & 0.0599312 & -0.1054211 \\
0.300 & -0.0030174 & 0.0786598 & 0.0524398 & -0.0731051 \\
0.400 & -0.0018307 & 0.0674226 & 0.0449484 & -0.0482791 \\
0.500 & -0.0010703 & 0.0561855 & 0.0374570 & -0.0296238 \\
0.600 & -0.0005856 & 0.0449484 & 0.0299656 & -0.0161272 \\
0.700 & -0.0002853 & 0.0337113 & 0.0224742 & -0.0069946 \\
0.800 & -0.0001110 & 0.0224742 & 0.0149828 & -0.0015907 \\
0.900 & -0.0000246 & 0.0112371 & 0.0074914 & 0.0005999 \\
1.000 & -0.0000001 & -0.0000000 & -0.0000000 & -0.0000000 \\
2.000 & -0.0009005 & -0.1123711 & -0.0749141 & -0.1035238 \\
3.000 & -0.0019699 & -0.2247422 & -0.1498281 & -0.2978541 \\
4.000 & -0.0027962 & -0.3371132 & -0.2247422 & -0.5317316 \\
5.000 & -0.0034227 & -0.4494843 & -0.2996562 & -0.7863922 \\
6.000 & -0.0039072 & -0.5618554 & -0.3745703 & -1.0533265 \\
7.000 & -0.0042908 & -0.6742265 & -0.4494843 & -1.3281138 \\
8.000 & -0.0046012 & -0.7865976 & -0.5243984 & -1.6082296 \\
9.000 & -0.0048572 & -0.8989686 & -0.5993124 & -1.8921265 \\
10.000 & -0.0050716 & -1.0113397 & -0.6742265 & -2.1788038 \\
\hline
\end{tabular}

Table 12: Dependence on $E^{I} / E$ for $\int_{Y} \boldsymbol{T}^{i j} d \boldsymbol{y}$ 
negligible with respect to $\varepsilon$. This is a priori a difficult task: the abstract error estimates results on FEM approximations depend on the regularity of the solution (see [23]). For instance, since we use $P 2$ finite elements, the regularity of $\boldsymbol{u}^{0}$ implies that for the norms in $L^{2}(\Omega)$ we have the estimate:

$$
\left\|\boldsymbol{u}_{0}^{\text {out }}-\boldsymbol{u}_{0}^{\text {out }}\right\|=\left\|\boldsymbol{u}_{h}^{0}-\boldsymbol{u}^{0}\right\| \leq C h^{3}
$$

However $\boldsymbol{u}^{\varepsilon}$ and $\boldsymbol{u}^{1}$ are not so regular that an analogous of (50) be valid. In any case it must be pointed out that for small values of $\varepsilon$ it is too expensive to choose discretization steps such that the approximation errors be negligible with respect to $\varepsilon$. For this reasons a judicious choice of feasible and reasonable meshes is required. In most of the computations we take a regular triangulation $\Omega_{h}$ of $\Omega$ with mesh-size $h=1 / 120$ (and 57600 elements P2-Lagrange) and a varying mesh for $\Omega_{h}^{\varepsilon}$ where the largest mesh-size is $h_{\varepsilon}=1 / 80$. In the case of holes, we get 32240 elements P2-Lagrange when $\varepsilon=0.05,54240$ when $\varepsilon=0.025$ and 78240 when $\varepsilon=0.0125$. In the case of elastic inclusions, we get 35440 elements P2-Lagrange when $\varepsilon=0.05,59200$ when $\varepsilon=0.025$ and 88480 when $\varepsilon=0.0125$.

The numerical results for the different situations (holes, elastic inclusions with $p=0$ and $p=1$ ) are given in tables 13,14 and 15. It appears immediately that in all situations $\left\|\boldsymbol{u}_{h}^{\varepsilon}-\boldsymbol{u}_{0, h}^{\text {out }}\right\| /\left\|\boldsymbol{u}_{h}^{\varepsilon}\right\| \approx \varepsilon$ and $\left\|\boldsymbol{u}_{h}^{\varepsilon}-\boldsymbol{u}_{1, h}^{\text {out }}\right\| /$ $\left\|\boldsymbol{u}_{h}^{\varepsilon}\right\| \approx \varepsilon^{m}$ with $m \gtrsim 1.5$.

\begin{tabular}{|c|l|l|}
\hline$\varepsilon$ & $\frac{\left\|\boldsymbol{u}_{h}^{\varepsilon}-\boldsymbol{u}_{0, h}^{\text {out }}\right\|_{L^{2}}}{\left\|\boldsymbol{u}_{h}^{\varepsilon}\right\|_{L^{2}}}$ & $\frac{\left\|\boldsymbol{u}_{h}^{\varepsilon}-\boldsymbol{u}_{1, h}^{\text {out }}\right\|_{L^{2}}}{\left\|\boldsymbol{u}_{h}^{\varepsilon}\right\|_{L^{2}}}$ \\
\hline 0.05 & 0.0224 & 0.0009025 \\
0.025 & 0.0112 & 0.0003187 \\
0.0125 & 0.00559 & 0.0001114 \\
\hline
\end{tabular}

Table 13: Relative errors : holes

Another peculiarity of the matched asymptotic expansion method is that the quality of the outer approximation increases far from the surface $\omega$ where the heterogeneities are concentrated. In order to test that $\boldsymbol{u}_{1, h}^{\text {out }}$ gives indeed a better approximation of $\boldsymbol{u}_{h}^{\varepsilon}$ far from the heterogeneities (and obviously far from the singularities of $\boldsymbol{u}_{h}^{\varepsilon}$ induced by the geometry of $\Omega$ or by the loading) we introduce for $\delta \geq 0$ the domains $\hat{\Omega}^{\text {out }}(\delta):=\left\{\boldsymbol{x}=\left(x_{1}, x_{2}\right) \in \Omega^{\varepsilon} ;\left|x_{1}\right| \geq \frac{\delta}{2}\right\}$. We then computed the relative errors norm in $\boldsymbol{L}^{2}\left(\hat{\Omega}^{\text {out }}(\delta)\right)$ for different values 


\begin{tabular}{|c|l|l|}
\hline$\varepsilon$ & $\frac{\left\|\boldsymbol{u}_{h}^{\varepsilon}-\boldsymbol{u}_{0, h}^{\text {out }}\right\|_{L^{2}}}{\left\|\boldsymbol{u}_{h}^{\varepsilon}\right\|_{L^{2}}}$ & $\frac{\left\|\boldsymbol{u}_{h}^{\varepsilon}-\boldsymbol{u}_{1, h}^{\text {out }}\right\|_{L^{2}}}{\left\|\boldsymbol{u}_{h}^{\varepsilon}\right\|_{L^{2}}}$ \\
\hline 0.05 & 0.00416 & 0.000206 \\
0.025 & 0.00206 & 0.0000781 \\
0.0125 & 0.00101 & 0.0000247 \\
\hline
\end{tabular}

Table 14: Relative errors : elastic inclusions with $p=0$ and $E^{I}=0.5 E$

\begin{tabular}{|c|l|l|}
\hline$\varepsilon$ & $\frac{\left\|\boldsymbol{u}_{h}^{\varepsilon}-\boldsymbol{u}_{0, h}^{\text {out }}\right\|_{L^{2}}}{\left\|\boldsymbol{u}_{h}^{\varepsilon}\right\|_{L^{2}}}$ & $\frac{\left\|\boldsymbol{u}_{h}^{\varepsilon}-\boldsymbol{u}_{1, h}^{\text {out }}\right\|_{L^{2}}}{\left\|\boldsymbol{u}_{h}^{\varepsilon}\right\|_{L^{2}}}$ \\
\hline 0.05 & 0.020022435 & 0.001825071 \\
0.025 & 0.010576063 & 0.000451827 \\
0.0125 & 0.005423596 & 0.000122152 \\
\hline
\end{tabular}

Table 15: Relative errors : elastic inclusions with $p=1$ and $E^{I}=0.5 E$

of $\varepsilon$ in the case of the holes and in the case of elastic inclusions with $p=0$ and $p=1$ (see tables 16, 17 and 18). The numerical results not only agree with this property of the matched asymptotic expansion method but also prove that if one takes the same domain $\hat{\Omega}^{\text {out }}(\delta)$ then the error due to the approximation decreases with $\varepsilon$ with an order $\gtrsim 1.5$ : see e.g. the values of $\delta / \varepsilon=1$, resp. $=2$, resp. $=4$ when $\varepsilon=0.05$, resp. $=0.025$, resp. $=0.0125$ or analogous triplet of values.

The correction of $\boldsymbol{u}^{0}$ by $\varepsilon \boldsymbol{u}^{1}$ is of order $\varepsilon$. One might then say that the need to calculate $\boldsymbol{u}^{1}$ depends on the precision with which one wishes to estimate $\boldsymbol{u}^{\varepsilon}$. However it is important to remember that $\boldsymbol{u}_{0}^{\text {out }}$ and $\boldsymbol{u}_{1}^{\text {out }}$ do not provide any information about inclusions and the approximation of the behaviour of $\boldsymbol{u}^{\varepsilon}$ in the layer $\Omega^{i n, \varepsilon}$ by the inner term $\boldsymbol{v}^{0}+\varepsilon \boldsymbol{v}^{1}$ requires the calculation of $\boldsymbol{u}^{1}$ to recover the uniqueness of $\boldsymbol{v}^{1}$ (see remark 2.2.1). Therefore the implementation and validation of the computation of $\boldsymbol{u}_{h}^{1}$ is necessary.

\subsection{Validation at the microscopic scale: the domain $\Omega_{h}^{i n}(\varepsilon)$}

Let us first remark that as shown in (16) $\boldsymbol{u}_{0, h}^{i n n}:=\boldsymbol{u}_{h}^{0}$ is independent from the inclusions. Hence this validation will be realized studying the relative error norms for $\boldsymbol{u}_{1, h}^{i n n}$ in a suitably defined domain $\Omega_{h}^{i n}(\varepsilon)$ whose thickness is defined by the function $\eta(\varepsilon)$ which is not a given datum and has to be 


\begin{tabular}{|c|c|c|c|c|c|}
\hline \multicolumn{2}{|r|}{$\varepsilon=0.05$} & \multicolumn{2}{|r|}{$\varepsilon=0.025$} & \multicolumn{2}{|c|}{$\varepsilon=0.0125$} \\
\hline \multirow{2}{*}{$\delta / \varepsilon$} & $\left.\left|\boldsymbol{u}_{h}^{\varepsilon}-\boldsymbol{u}_{1 h}^{\text {out }}\right|\right|_{L^{2}}$ & & $\left.\left|\boldsymbol{u}_{h}^{\varepsilon}-\boldsymbol{u}_{1 h}^{\text {out }}\right|\right|_{L^{2}}$ & \multirow{2}{*}{$\delta / \varepsilon$} & $\left\|\boldsymbol{u}_{h}^{\varepsilon}-\boldsymbol{u}_{1 h}^{\text {out }}\right\|_{L^{2}}$ \\
\hline & 10 & $\partial / \varepsilon$ & 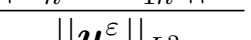 & & $\left\|\boldsymbol{u}_{h}^{\varepsilon}\right\|_{L^{2}}$ \\
\hline 0.0 & $\frac{\left\|\boldsymbol{\omega}_{h}\right\| L^{2}}{0.000902513}$ & 0.0 & $\frac{\left\|\boldsymbol{u}_{h}\right\| L^{2}}{0.000318681}$ & 0.0 & 0.000111381 \\
\hline 1.0 & 0.000841890 & 2.0 & 0.000296530 & 2.0 & 0.000106239 \\
\hline 1.2 & 0.000837274 & 3.0 & 0.000295194 & 4.0 & 0.000104731 \\
\hline 1.4 & 0.000834825 & 4.0 & 0.000294175 & 6.0 & 0.000103745 \\
\hline 1.6 & 0.000833395 & 5.0 & 0.000293475 & 100 & 0.000102848 \\
\hline 1.8 & 0.000832471 & 6.0 & 0.000293108 & 10.0 & 0.000102221 \\
\hline 2.0 & 0.000831834 & 7.0 & 0.000292848 & 12.0 & 0.000101890 \\
\hline 3.0 & 0.000830932 & 8.0 & 0.000292778 & 16.0 & 0.000101313 \\
\hline & & & & 20.0 & 0.000101085 \\
\hline
\end{tabular}

Table 16: Evolution of the exterior approximation in $\hat{\Omega}^{\text {out }}(\delta)$ : holes

\begin{tabular}{|c|c|}
\hline \multicolumn{2}{|c|}{$\varepsilon=0.05$} \\
\hline$\delta / \varepsilon$ & $\frac{\left\|\boldsymbol{u}_{h}^{\varepsilon}-\boldsymbol{u}_{1 h}^{\text {out }}\right\|_{L^{2}}}{\left\|\boldsymbol{u}_{h}^{\varepsilon}\right\|_{L^{2}}}$ \\
\hline 0.0 & 0.000205545 \\
1.0 & 0.000189485 \\
1.2 & 0.000189295 \\
1.4 & 0.000188847 \\
1.6 & 0.000188449 \\
1.8 & 0.000188183 \\
2.0 & 0.000187997 \\
3.0 & 0.000187725 \\
4.0 & 0.000187797 \\
\hline
\end{tabular}

\begin{tabular}{|c|c|}
\hline \multicolumn{2}{|c|}{$\varepsilon=0.025$} \\
\hline$\delta / \varepsilon$ & $\frac{\left\|\boldsymbol{u}_{h}^{\varepsilon}-\boldsymbol{u}_{1 h}^{\text {out }}\right\|_{L^{2}}}{\left\|\boldsymbol{u}_{h}^{\varepsilon}\right\|_{L^{2}}}$ \\
\hline 0.0 & 0.000078085 \\
1.0 & 0.000072545 \\
2.0 & 0.000071793 \\
3.0 & 0.000071304 \\
4.0 & 0.000070938 \\
5.0 & 0.000070701 \\
6.0 & 0.000070564 \\
7.0 & 0.000070465 \\
8.0 & 0.000070419 \\
9.0 & 0.000070414 \\
10.0 & 0.000070431 \\
\hline
\end{tabular}

\begin{tabular}{|c|c|}
\hline \multicolumn{2}{|c|}{$\varepsilon=0.0125$} \\
\hline$\delta / \varepsilon$ & $\frac{\left\|\boldsymbol{u}_{h}^{\varepsilon}-\boldsymbol{u}_{1 h}^{\text {out }}\right\|_{L^{2}}}{\left\|\boldsymbol{u}_{h}^{\varepsilon}\right\|_{L^{2}}}$ \\
\hline 0.0 & 0.000024692 \\
2.0 & 0.000021741 \\
4.0 & 0.000021028 \\
6.0 & 0.000020559 \\
8.0 & 0.000020086 \\
10.0 & 0.000019666 \\
12.0 & 0.000019367 \\
14.0 & 0.000019129 \\
16.0 & 0.000018938 \\
20.0 & 0.000018588 \\
\hline
\end{tabular}

Table 17: Evolution of the exterior approximation in $\hat{\Omega}^{\text {out }}(\delta)$ : elastic inclusions $p=0$, $E^{I}=0.5 E$ 


\begin{tabular}{|c|c|c|c|c|c|}
\hline \multicolumn{2}{|r|}{$\varepsilon=0.05$} & \multicolumn{2}{|c|}{$\varepsilon=0.025$} & \multicolumn{2}{|c|}{$\varepsilon=0.0125$} \\
\hline \multirow{2}{*}{$\delta / \varepsilon$} & $\left\|\boldsymbol{u}_{h}^{\varepsilon}-\boldsymbol{u}_{1 h}^{\text {out }}\right\|_{L^{2}}$ & $\delta / \varepsilon$ & $\left\|\boldsymbol{u}_{h}^{\varepsilon}-\boldsymbol{u}_{1 h}^{\text {out }}\right\|_{L^{2}}$ & $\delta / \varepsilon$ & $\left\|\boldsymbol{u}_{h}^{\varepsilon}-\boldsymbol{u}_{1 h}^{\text {out }}\right\| \|_{L^{2}}$ \\
\hline & $\left\|\boldsymbol{u}_{h}^{\varepsilon}\right\|_{L^{2}}$ & $0 / \mathrm{c}$ & $\left\|\boldsymbol{u}_{h}^{\varepsilon}\right\|_{L^{2}}$ & $0 / \mathrm{c}$ & $\left\|\boldsymbol{u}_{h}^{\varepsilon}\right\|_{L^{2}}$ \\
\hline 0.0 & 0.001825071 & 0.0 & 0.000451827 & 0.0 & 0.000122152 \\
\hline 1.0 & 0.001759737 & 1.0 & 0.000422243 & 2.0 & 0.000109975 \\
\hline 1.2 & 0.001757144 & 2.0 & 0.000417829 & 4.0 & 0.000108002 \\
\hline 1.4 & 0.001755302 & 3.0 & 0.000415797 & 6.0 & 0.000106582 \\
\hline 1.6 & 0.001753779 & 4.0 & 0.000413844 & 8.0 & 0.000105119 \\
\hline 1.8 & 0.001752386 & 5.0 & 0.000412015 & 10.0 & 0.000103909 \\
\hline 2.0 & 0.001751041 & 6.0 & 0.000410613 & 12.0 & 0.000102889 \\
\hline 3.0 & 0.001747021 & 7.0 & 0.000408883 & 14.0 & 0.000101912 \\
\hline 4.0 & 0.001741251 & 8.0 & 0.000407363 & 16.0 & 0.000101275 \\
\hline 5.0 & 0.001736350 & 10.0 & 0.000404803 & 20.0 & 0.000099688 \\
\hline
\end{tabular}

Table 18: Evolution of the exterior approximation in $\hat{\Omega}^{\text {out }}(\delta)$ : elastic inclusions $p=1$ and $E^{I}=0.5 E$

computationally evaluated. As has been previously mentioned at the beginning of sect. 4.3 , in order to compute these relative error norms one must determine the nodal values of $\boldsymbol{u}_{1, h}^{i n n}:=\boldsymbol{v}_{h}^{0}+\varepsilon \boldsymbol{v}_{h}^{1}$ on the mesh $\Omega_{h}^{\varepsilon}$ where the numerical reference solution $\boldsymbol{u}_{h}^{\varepsilon}$ is defined. We use the same meshes as for the macroscopic scale validation. In order to numerically evaluate $\eta(\varepsilon)$ at first we compute for increasing values of $\eta>0$ the relative errors $\frac{\left\|\boldsymbol{u}_{h}^{\varepsilon}-\boldsymbol{u}_{1 h}^{i n n}\right\|_{L^{2}}}{\left\|\boldsymbol{u}_{h}^{\varepsilon}\right\|_{L^{2}}}$ in the domain $\Omega^{\eta, \varepsilon}=\left\{\boldsymbol{x}=\left(x_{1}, x_{2}\right) \in \Omega^{\varepsilon}:\left|x_{1}\right|<\frac{\eta}{2}\right\}$. Then $\eta(\varepsilon)$ will be in the interval between the value of $\eta$ such that this relative errors is minimum and the next value of $\eta$. In the case of holes the results are given in table 19. We also give in table 20 the estimated value of $\eta(\varepsilon)$ and of $\eta(\varepsilon) / \varepsilon$. For elastic inclusions with $p=0$ and $E^{I}=0.5 E$ the analogous results are given in tables 21 and 22. All these results are in agreement with the asymptotic assumptions, in particular with (3). 


\begin{tabular}{|c|c|}
\hline \multicolumn{2}{|c|}{$\varepsilon=0.05$} \\
\hline$\eta / \varepsilon$ & $\frac{\left\|\boldsymbol{u}_{h}^{\varepsilon}-\boldsymbol{u}_{1 h}^{i n n}\right\|_{L^{2}}}{\left\|\boldsymbol{u}_{h}^{\varepsilon}\right\|_{L^{2}}}$ \\
\hline 1.2 & 0.002774 \\
1.4 & 0.002751 \\
1.6 & 0.002780 \\
\hline
\end{tabular}

\begin{tabular}{|c|c|}
\hline \multicolumn{2}{|c|}{$\varepsilon=0.025$} \\
\hline$\eta / \varepsilon$ & $\frac{\left\|\boldsymbol{u}_{h}^{\varepsilon}-\boldsymbol{u}_{1 h}^{i n n}\right\|_{L^{2}}}{\left\|\boldsymbol{u}_{h}^{\varepsilon}\right\|_{L^{2}}}$ \\
\hline 1.2 & 0.001036958 \\
1.4 & 0.001005650 \\
1.6 & 0.001000810 \\
1.8 & 0.001002316 \\
2.0 & 0.001016178 \\
\hline
\end{tabular}

\begin{tabular}{|c|c|}
\hline \multicolumn{2}{|c|}{$\varepsilon=0.0125$} \\
\hline$\eta / \varepsilon$ & $\frac{\left\|\boldsymbol{u}_{h}^{\varepsilon}-\boldsymbol{u}_{1 h}^{i n n}\right\|_{L^{2}}}{\left\|\boldsymbol{u}_{h}^{\varepsilon}\right\|_{L^{2}}}$ \\
\hline 1.2 & 0.000414496 \\
1.4 & 0.000408768 \\
1.6 & 0.000400531 \\
1.8 & 0.000394518 \\
2.0 & 0.000391273 \\
2.2 & 0.000404875 \\
2.4 & 0.000408182 \\
\hline
\end{tabular}

Table 19: Evolution of the interior approximation in $\Omega^{\eta, \varepsilon}$ : holes

\begin{tabular}{|c|c|c|}
\hline$\varepsilon=0.05$ & $0.07 \leq \eta(\varepsilon) \leq 0.08$ & $1.4 \leq \eta(\varepsilon) / \varepsilon \leq 1.6$ \\
$\varepsilon=0.025$ & $0.04 \leq \eta(\varepsilon) \leq 0.045$ & $1.6 \leq \eta(\varepsilon) / \varepsilon \leq 1.8$ \\
$\varepsilon=0.0125$ & $0.025 \leq \eta(\varepsilon) \leq 0.0275$ & $2 \leq \eta(\varepsilon) / \varepsilon \leq 2.2$ \\
\hline
\end{tabular}

Table 20: Evolution of $\eta(\varepsilon)$ and of $\eta(\varepsilon) / \varepsilon$ with $\varepsilon$ : holes

\begin{tabular}{|c|c||c|c|}
\hline \multicolumn{2}{|c|}{$\varepsilon=0.05$} \\
\cline { 2 - 3 }$\eta / \varepsilon$ & $\frac{\left\|\boldsymbol{u}_{h}^{\varepsilon}-\boldsymbol{u}_{1 h}^{i n n}\right\|_{L^{2}}}{\left\|\boldsymbol{u}_{h}^{\varepsilon}\right\|_{L^{2}}}$ \\
\cline { 2 - 3 } & 0.0006462 \\
1.0 & 0.0006605 \\
1.2 & $\frac{\left\|\boldsymbol{u}_{h}^{\varepsilon}-\boldsymbol{u}_{1 h}^{i n n}\right\|_{L^{2}}}{\left\|\boldsymbol{u}_{h}^{\varepsilon}\right\|_{L^{2}}}$ \\
1.4 & 0.0006968 \\
1.0 & 0.0002882 \\
1.2 & 0.0002837 \\
1.4 & 0.0002848 \\
1.6 & 0.0002917 \\
\hline
\end{tabular}

\begin{tabular}{|c|c|}
\hline \multicolumn{2}{|c|}{$\varepsilon=0.0125$} \\
\hline$\eta / \varepsilon$ & $\frac{\left\|\boldsymbol{u}_{h}^{\varepsilon}-\boldsymbol{u}_{1 h}^{i n n}\right\|_{L^{2}}}{\left\|\boldsymbol{u}_{h}^{\varepsilon}\right\|_{L^{2}}}$ \\
\hline 1.2 & 0.0001341 \\
1.4 & 0.0001330 \\
1.6 & 0.0001312 \\
1.8 & 0.0001299 \\
2.0 & 0.0001302 \\
\hline
\end{tabular}

Table 21: Evolution of the interior approximation in $\Omega^{\eta, \varepsilon}$; elastic inclusions: $p=0$, $E^{I}=0.5 E$

\begin{tabular}{|c|c|c|}
\hline$\varepsilon=0.05$ & $0.05 \leq \eta(\varepsilon) \leq 0.06$ & $1 . \leq \eta(\varepsilon) / \varepsilon \leq 1.2$ \\
$\varepsilon=0.025$ & $0.03 \leq \eta(\varepsilon) \leq 0.035$ & $1.2 \leq \eta(\varepsilon) / \varepsilon \leq 1.4$ \\
$\varepsilon=0.0125$ & $0.0225 \leq \eta(\varepsilon) \leq 0.025$ & $1.8 \leq \eta(\varepsilon) / \varepsilon \leq 2$ \\
\hline
\end{tabular}

Table 22: Evolution of $\eta(\varepsilon)$ and of $\eta(\varepsilon) / \varepsilon$ with $\varepsilon$; elastic inclusions: $p=0, E^{I}=0.5 E$ 


\subsection{Validation at the microscopic scale: the stress field near the inclusions}

Important qualitative and quantitative informations on the behaviour of the structure are given by the stress field, in particular its "concentration" in a neighbourhood of every inclusion. As suggested by Nguetseng and Sànchez-Palencia [1], the application of the multi-scale matched asymptotic expansion method must allow to obtain those informations. In order to numerically verify this suggestion it is important to compare $\boldsymbol{\sigma}_{h}^{\varepsilon}$ and $\boldsymbol{\tau}_{\boldsymbol{h}}{ }^{0}$ near the inclusions. In the following this comparison is done for $\varepsilon=0.0125$, for both holes and elastic inclusions with $p=0$. We consider 4 inclusions $\mathcal{I}_{k}^{\varepsilon}$, $k=1,2,3$ and 4 , located in $\Omega^{\varepsilon}$ by their center of coordinates $\left(x_{1}=0, x_{2}^{k}\right)$ with $x_{2}^{k}=-20 \varepsilon,-\varepsilon, 10 \varepsilon$ and $30 \varepsilon$. Let $\boldsymbol{\sigma}_{h}^{\varepsilon, V M}$ (resp. $\boldsymbol{\tau}_{h}^{0, V M}$ ), denote the equivalent Von Mises stress of $\boldsymbol{\sigma}_{h}^{\varepsilon}$ (resp. $\boldsymbol{\tau}_{h}{ }^{0}$ ). We plot the isovalues of $\boldsymbol{\sigma}_{h}^{\varepsilon, V M}$ in a neighbourhood of each inclusion and we compare them to the isovalues of $\boldsymbol{\tau}_{h}^{0, V M}$. The results in the case of holes are given in fig. 11, 12, 13 and 14; in the case of elastic inclusions with $p=0$ and $E^{I} / E=4$ in fig.15, 16, 17 and 18.
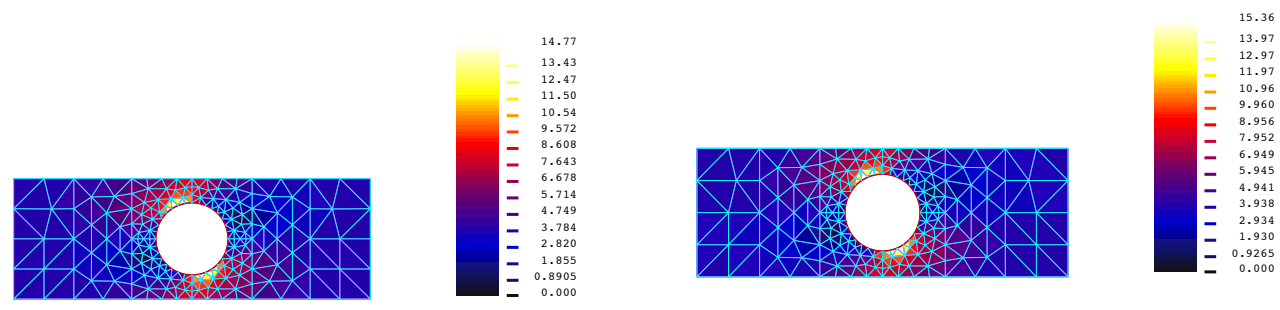

Figure 11: Comparison of $\boldsymbol{\sigma}_{h}^{\varepsilon, V M}$ (left) and $\boldsymbol{\tau}_{h}^{0, V M}$ (right) in a neighbourhood of $\mathcal{I}_{1}^{\varepsilon}$

\section{Concluding remarks}

We have numerically validate a recently proposed [2], [3] variant of the multi-scale matched asymptotic expansion method proposed by Nguetseng and Sànchez-Palencia in a pioneering paper [1]. In opposition to [1] we are investigating not only the local but also the global behavior of the structure. The zero order problems do not consider the heterogeneities and hence give no 


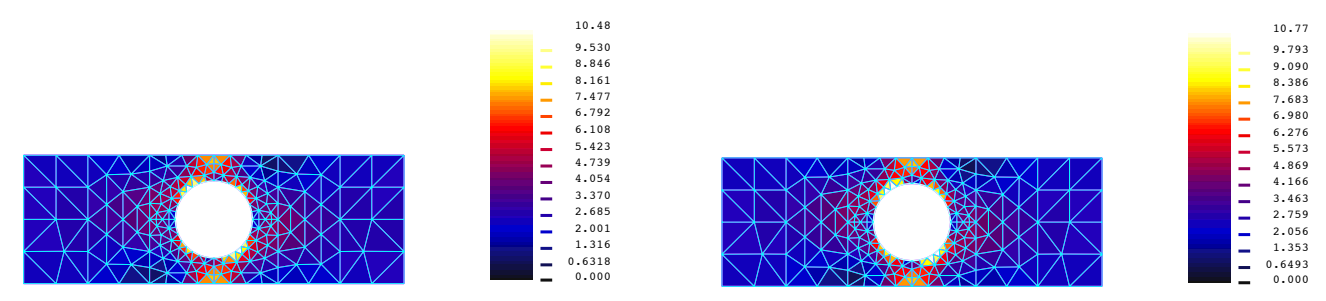

Figure 12: Comparison of $\boldsymbol{\sigma}_{h}^{\varepsilon, V M}$ (left) and $\boldsymbol{\tau}_{h}^{0, V M}$ (right) in a neighbourhood of $\mathcal{I}_{2}^{\varepsilon}$
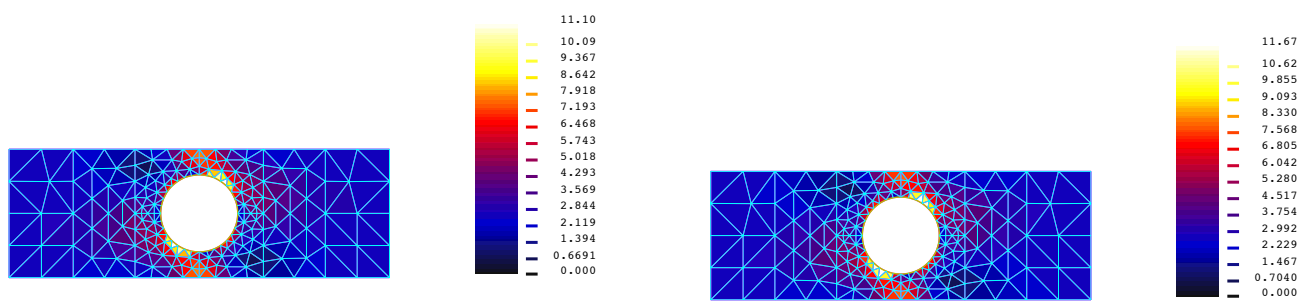

Figure 13: Comparaison of $\boldsymbol{\sigma}_{h}^{\varepsilon, V M}$ (left) and $\boldsymbol{\tau}_{h}^{0, V M}$ (right) in a neighbourhood of $\mathcal{I}_{3}^{\varepsilon}$
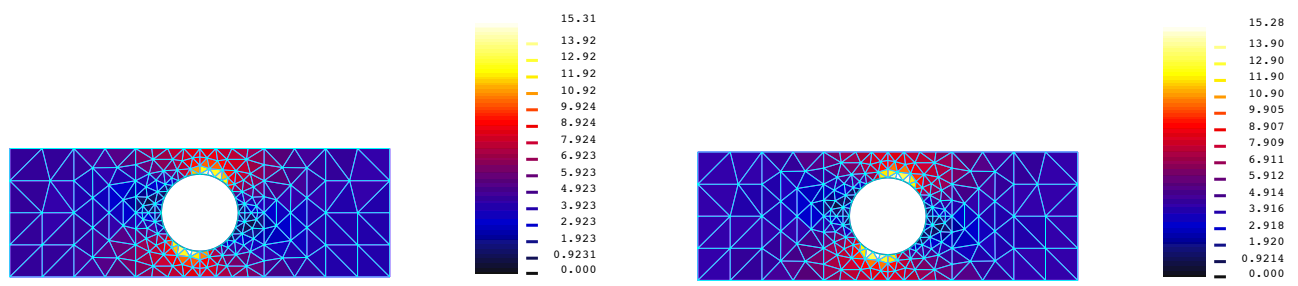

Figure 14: Comparaison of $\boldsymbol{\sigma}_{h}^{\varepsilon, V M}$ (left) and $\boldsymbol{\tau}_{h}^{0, V M}$ (right) in a neighbourhood of $\mathcal{I}_{4}^{\varepsilon}$ 


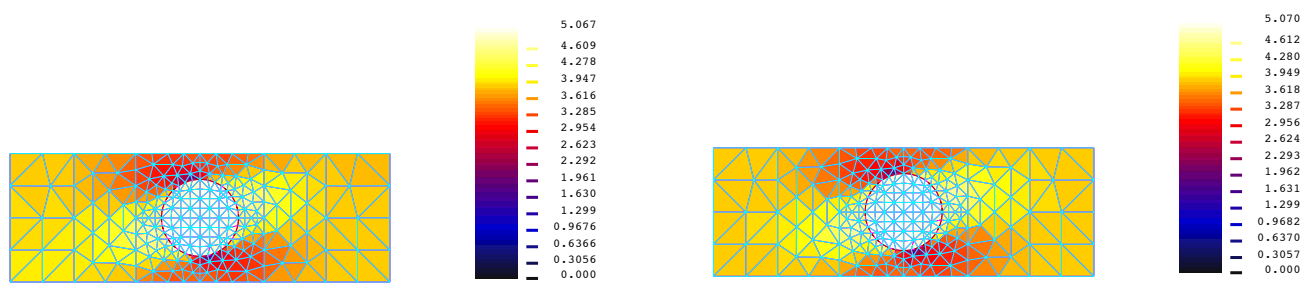

Figure 15: Comparison of $\boldsymbol{\sigma}_{h}^{\varepsilon, V M}$ (left) and $\boldsymbol{\tau}_{h}^{0, V M}$ (right) in a neighbourhood of $\mathcal{I}_{1}^{\varepsilon}$, elastic inclusion with $E^{I} / E=4$

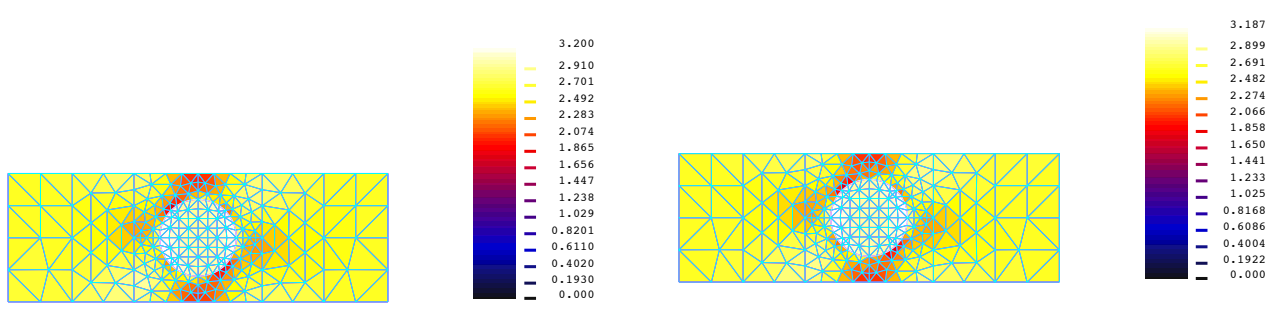

Figure 16: Comparison of $\boldsymbol{\sigma}_{h}^{\varepsilon, V M}$ (left) and $\boldsymbol{\tau}_{h}^{0, V M}$ (right) in a neighbourhood of $\mathcal{I}_{2}^{\varepsilon}$, elastic inclusion with $E^{I} / E=4$

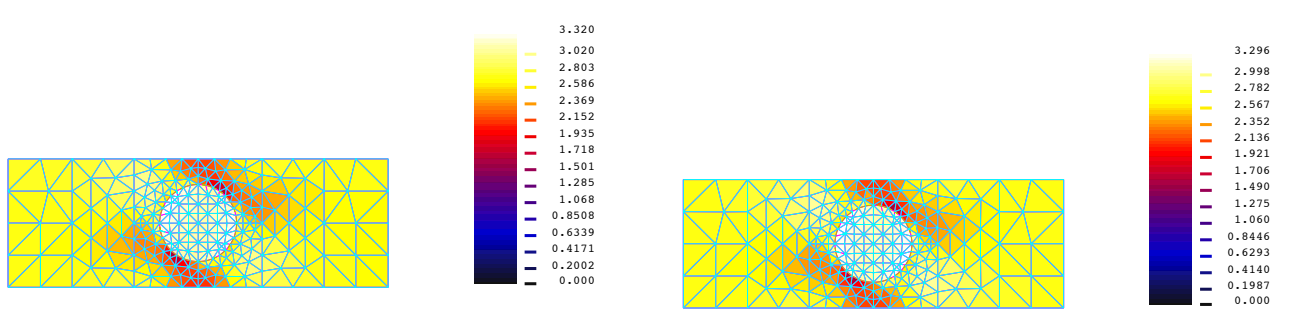

Figure 17: Comparaison of $\boldsymbol{\sigma}_{h}^{\varepsilon, V M}$ (left) and $\boldsymbol{\tau}_{h}^{0, V M}$ (right) in a neighbourhood of $\mathcal{I}_{3}^{\varepsilon}$, elastic inclusion with $E^{I} / E=4$ 


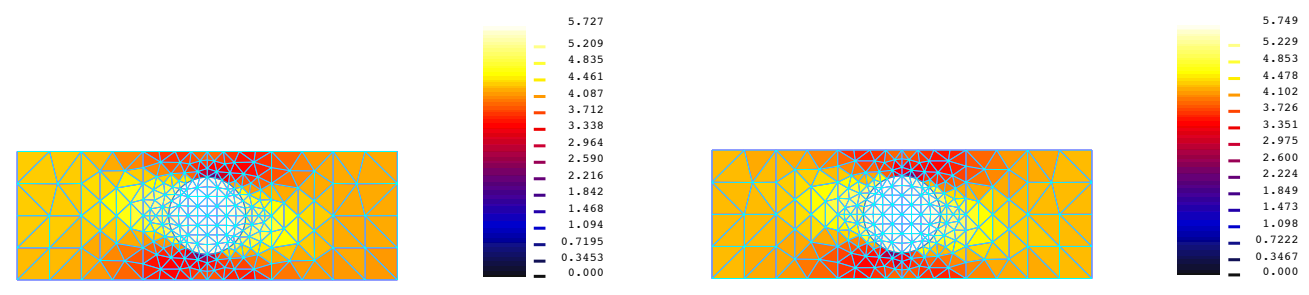

Figure 18: Comparison of $\boldsymbol{\sigma}_{h}^{\varepsilon, V M}$ (left) and $\boldsymbol{\tau}_{h}^{0, V M}$ (right) in a neighbourhood of $\mathcal{I}_{4}^{\varepsilon}$, elastic inclusion with $E^{I} / E=4$

information on the microscopic behaviour near the heterogeneities. However the first order problems allow to obtain the necessary informations in order to reconstruct displacement and stress fields around the heterogeneities. We have implemented a robust numerical algorithm which combines matched asymptotic expansions and domain decomposition obtaining a tool to effectively solve multi-scale problems with a large number of heterogeneities. Let us also emphasize that the numerical experiments agree with the assumptions at the basis of the formal multi-scale matched asymptotic expansion method.

As in the classical homogenization [4], [5], [6], [7] the coefficients useful for the first order problems can be easily computed once for all as solutions of cell problems. Indeed these cell problems do not depend on the forces and displacements imposed on the whole structure, but only on the geometry of the heterogeneities and on the material properties. Many numerical tests have been done in order not only to prove the robustness of the computations but also to study the dependence of these coefficients on the geometric and materials parameters: for instance the diameter in the case of the holes and the relative strength for elastic inclusions.

It is also important to put in evidence that the zeroth and the first order approximations are computed on a structure without the heterogeneities using a coarse mesh easy to obtain. The numerical validation proves that the global and local information obtained is as good as the one obtained on the real structure with the heterogeneities on a very fine mesh which is difficult to realize, at a better computational cost. 


\section{Appendix}

In order to avoid inessential technicalities we consider the following simplified form of the first order problem (32) where we assume that $\omega$ is at least of class $\mathcal{C}^{2}$, that $\omega \cap \partial \Omega=\emptyset$ and that $\partial \Omega_{u}=\partial \Omega$ :

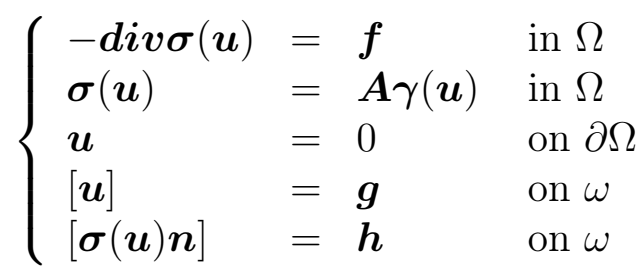

where $\boldsymbol{f} \in \boldsymbol{L}^{2}(\Omega), \boldsymbol{g} \in \boldsymbol{H}^{-1 / 2}(\omega)$ and $\boldsymbol{h} \in \boldsymbol{H}^{-3 / 2}(\omega)$ denote the gap in displacements and in normal stresses on $\omega$. These data are known and can be computed once the zero order problem is solved. Let us first remark that the solution $\boldsymbol{u}$ of (43) does not belong to $\boldsymbol{H}^{1}(\Omega)$. More precisely, let be $\Omega_{1}:=\Omega^{+}, \Omega_{2}:=\Omega^{-}$and :

$$
\boldsymbol{Z}:=\left\{\boldsymbol{z} \in \boldsymbol{L}^{2}(\Omega) ; \operatorname{div}\left(\boldsymbol{A} \boldsymbol{\gamma}\left(\left.\boldsymbol{z}\right|_{\Omega_{i}}\right) \in \boldsymbol{L}^{2}\left(\Omega_{i}\right)\right\}\right.
$$

equipped with the natural graph norm. Then $\boldsymbol{u} \in \boldsymbol{Z}$. Since $\left.\left(\left.\boldsymbol{z}\right|_{\Omega_{i}}\right)\right|_{\omega}$ and $\left(\left.\left(\boldsymbol{A} \boldsymbol{\gamma}\left(\left.\boldsymbol{z}\right|_{\Omega_{i}}\right) \boldsymbol{n}\right)\right|_{\omega}\right.$ cannot have a classical meaning for $\boldsymbol{u} \in \boldsymbol{Z}$ the transmission conditions $[\boldsymbol{u}]=\mathcal{G}_{\boldsymbol{d}}$ and $[\boldsymbol{\sigma}(\boldsymbol{u}) \boldsymbol{n}]=\mathcal{G}_{\boldsymbol{n} \boldsymbol{S}}$ on $\omega$ have to be taken in a weak sense adapting the methods of Lions-Magenes [17]. Let us consider separately $\Omega_{1}$ and $\Omega_{2}$ and with an obvious notation $\boldsymbol{Z}\left(\Omega_{i}\right)$ and let us assume that the elasticity tensor $\mathbf{A}$ is constant on each $\Omega_{i}$. We drop the index $i$ since the reasoning is the same for both cases. As in distribution theory $\left.\boldsymbol{z}\right|_{\omega}$ and $\left.(\boldsymbol{A} \gamma(\boldsymbol{z}) \boldsymbol{n})\right|_{\omega}$ will be defined by duality.

Theorem 1. The space $\mathcal{C}^{\infty}\left(\bar{\Omega} ; \mathbf{R}^{3}\right)$ is dense in $\boldsymbol{Z}(\Omega)$.

Proof. Let be $\mathbf{u} \longrightarrow \mathbf{L}(\mathbf{u})$ a linear and continuous form on $\boldsymbol{Z}(\Omega)$; since $\boldsymbol{L}^{2}(\Omega)$ is an Hilbert space there exist $\Psi, \Phi \in \boldsymbol{L}^{2}(\Omega)$ such that:

$$
\mathbf{L}(\mathbf{u})=\int_{\Omega} \Psi \mathbf{u} d x+\int_{\Omega} \Phi \operatorname{div} \boldsymbol{A} \gamma(\boldsymbol{u}) d x
$$

To conclude it is enough to prove that if

$$
\mathbf{L}(\theta)=0 \text { for all } \theta \in \mathcal{C}^{\infty}\left(\bar{\Omega} ; \mathbf{R}^{3}\right)
$$


then $\mathbf{L}(\mathbf{u})=0$ for all $\mathbf{u} \in \boldsymbol{Z}(\Omega)$. Since every $\theta \in \mathcal{C}^{\infty}\left(\bar{\Omega} ; \mathbf{R}^{3}\right)$ is the restriction to $\Omega$ of (at least) one $\Theta \in \mathcal{D}\left(\mathbf{R}^{3} ; \mathbf{R}^{3}\right)$ then we can write (54) a s:

$$
\mathbf{L}(\theta)=\int_{\mathbf{R}^{3}} \tilde{\Psi} \Theta d x+\int_{\mathbf{R}^{3}} \tilde{\Phi} \operatorname{div} \boldsymbol{A} \gamma(\Theta) d x=0
$$

where $\tilde{\Psi}, \tilde{\Phi} \in \boldsymbol{L}^{2}\left(\mathbf{R}^{3}\right)$ are the extensions with 0 outside $\Omega$ of $\Psi, \Phi \in \boldsymbol{L}^{2}(\Omega)$. Equation (55) means that in the distributions sense on $\mathbf{R}^{3}$ one has

$$
\operatorname{div} \boldsymbol{A} \gamma(\tilde{\Phi})=-\tilde{\Psi}
$$

and hence (local regularity of elliptic systems) that $\tilde{\Phi} \in \boldsymbol{H}^{2}(\tilde{\Omega})$ for any bounded $\tilde{\Omega} \supset \bar{\Omega}$. This means that indeed $\Phi \in \boldsymbol{H}_{0}^{2}(\Omega)$ and so integrating by parts (53) we get thanks to (55) $\mathbf{L}(\mathbf{u})=\int_{\Omega}(\operatorname{div} \boldsymbol{A} \gamma(\Phi)+\Psi) \mathbf{u} d x=0$.

Before to state and prove the fundamental result we recall the following lemma.

Lemma 1. The map $\mathfrak{t r}:=\mathbf{v} \longrightarrow\left(\left.\mathbf{v}\right|_{\omega},\left.(\boldsymbol{A} \gamma(\boldsymbol{v}) \boldsymbol{n})\right|_{\omega}\right)$ is linear, continuous and onto from $\boldsymbol{H}^{2}(\Omega)$ onto $\boldsymbol{H}^{3 / 2}(\omega) \times \boldsymbol{H}^{1 / 2}(\omega)$ and admits a non-unique linear right-inverse $\mathfrak{R}$; moreover $\operatorname{ker}(\mathfrak{t} \mathfrak{r})=\boldsymbol{H}_{0}^{2}(\Omega)$

The lemma is a particular case of a more general result on the so-called Cauchy data proved by Grubb, see e.g. [24] and the bibliography therein.

Theorem 2. The trace map $\mathfrak{t r}: \mathbf{u} \longrightarrow \mathfrak{t r}(\mathbf{u}):=\left(\left.\mathbf{u}\right|_{\omega},\left.(\boldsymbol{A} \boldsymbol{\gamma}(\boldsymbol{u}) \boldsymbol{n})\right|_{\omega}\right)$ defined on $\mathcal{C}^{\infty}\left(\bar{\Omega} ; \mathbf{R}^{3}\right)$ can be extended by continuity to a linear and continuous map, denoted $\mathfrak{T} \mathfrak{R}$, from $\boldsymbol{Z}(\Omega)$ into $\boldsymbol{H}^{-1 / 2}(\omega) \times \boldsymbol{H}^{-3 / 2}(\omega)$.

Proof. Let be given $(\eta, \varrho) \in \boldsymbol{H}^{3 / 2}(\omega) \times \boldsymbol{H}^{1 / 2}(\omega)$ and let be $\mathbf{v}(\eta, \varrho):=$ $\mathfrak{R}(-\eta, \varrho)$ where $\mathfrak{R}$ is one linear right inverse. Let now define on $\boldsymbol{Z}(\Omega) \times$ $\left(\boldsymbol{H}^{3 / 2}(\omega) \times \boldsymbol{H}^{1 / 2}(\omega)\right)$ the bilinear and bi-continuous form:

$$
L(\mathbf{u},(\eta, \varrho))=\int_{\Omega} \mathbf{u d i v} \boldsymbol{A} \boldsymbol{\gamma}(\mathbf{v}) d x-\int_{\Omega} \operatorname{div} \boldsymbol{A} \boldsymbol{\gamma}(\mathbf{u}) \mathbf{v} d x
$$

Let us first notice that $L(\mathbf{u},(\eta, \varrho))$ is well defined since it does not depend on the particular right inverse $\mathfrak{R}$ chosen. Indeed if $\mathbf{v}(\eta, \varrho)$ and $\mathbf{v}_{1}(\eta, \varrho)$ correspond to two different right inverses then $\left(\mathbf{v}(\eta, \varrho)-\mathbf{v}_{1}(\eta, \varrho)\right) \in \boldsymbol{H}_{0}^{2}(\Omega)$. The density of $\mathcal{D}\left(\mathbf{R}^{3} ; \mathbf{R}^{3}\right)$ in $\boldsymbol{H}_{0}^{2}(\Omega)$ and (57) imply the result. It is also 
obvious that $L(\mathbf{u},(\eta, \varrho))$ is bi-continuous and hence there exists a linear and continuous map $\mathfrak{T} \mathfrak{R}$, from $\boldsymbol{Z}(\Omega)$ to $\boldsymbol{H}^{-1 / 2}(\omega) \times \boldsymbol{H}^{-3 / 2}(\omega)$ such that

$$
L(\mathbf{u},(\eta, \varrho))=<\mathfrak{T} \mathfrak{R u},(\eta, \varrho)>
$$

where $<\bullet, \bullet>$ denotes the duality between $\boldsymbol{H}^{-1 / 2}(\omega) \times \boldsymbol{H}^{-3 / 2}(\omega)$ and $\boldsymbol{H}^{1 / 2}(\omega) \times \boldsymbol{H}^{3 / 2}(\omega)$. When $\mathbf{u} \in \mathcal{C}^{\infty}\left(\bar{\Omega} ; \mathbf{R}^{3}\right)$ then the Green formula, (57) and (58) imply that $\mathfrak{T} \mathfrak{R u}=\left(\left.\mathbf{u}\right|_{\omega},\left.(\boldsymbol{A} \boldsymbol{\gamma}(\boldsymbol{u}) \boldsymbol{n})\right|_{\omega}\right)$. Hence Theorem 1 implies the result.

Remark 1: Let us explicitly remark that the generic form of the first order problem (32) implies that $\mathbf{u} \in \boldsymbol{Z}_{0}:=\left\{\boldsymbol{z} \in \boldsymbol{L}^{2}(\Omega) ; \operatorname{div}\left(\boldsymbol{A} \boldsymbol{\gamma}\left(\left.\boldsymbol{z}\right|_{\Omega_{i}}\right)=0\right)\right\}$; .

\section{References}

[1] N. Nguetseng, E. Sánchez-Palencia, Stress concentration for defects distributed near a surface, in: Local effects in the analysis of structures (Cachan, 1984), Vol. 12 of Stud. Appl. Mech., Elsevier, Amsterdam, 1985, pp. 55-74.

[2] G. Geymonat, S. Hendili, F. Krasucki, M. Vidrascu, The matched asymptotic expansion for the computation of the effective behavior of an elastic structure with a thin layer of holes, International Journal for Multiscale Computational Engineering 9 (5) (2011) 529-542. doi:10.1615/IntJMultCompEng.v9.i5.

URL http://hal .inria.fr/inria-00540992

[3] G. Geymonat, S. Hendili, F. Krasucki, M. Vidrascu, Matched asymptotic expansion method for an homogenized interface model., Math. Models Methods Appl. Sci.doi:10.1142/S0218202513500607.

URL http://hal . archives-ouvertes.fr/hal-00757005

[4] A. Bensoussan, J.-L. Lions, G. Papanicolaou, Asymptotic analysis for periodic structures, AMS Chelsea Publishing, Providence, RI, 2011, corrected reprint of the 1978 original [MR0503330].

[5] O. A. Oleinik, A. S. Shamaev, G. A. Yosifian, Mathematical problems in elasticity and homogenization, Vol. 26 of Studies in Mathematics and its Applications, North-Holland Publishing Co., Amsterdam, 1992. 
[6] D. Cioranescu, P. Donato, An introduction to homogenization, Vol. 17 of Oxford Lecture Series in Mathematics and its Applications, The Clarendon Press Oxford University Press, New York, 1999.

[7] E. Sánchez-Palencia, Nonhomogeneous media and vibration theory, Vol. 127 of Lecture Notes in Physics, Springer-Verlag, Berlin, 1980.

[8] A.-L. Bessoud, F. Krasucki, G. Michaille, Multi-materials with strong interface: Variational modelings, Asymptotic Analysis 61 (2009) 1-19. doi:10.3233/ASY-2008-0903.

URL http://hal .archives-ouvertes.fr/hal-00790347

[9] G. Geymonat, S. Hendili, F. Krasucki, M. Serpilli, M. Vidrascu, Asymptotic expansions and domain decomposition, in: 21st International Conference on Domain Decomposition Methods, Rennes, France, 2013.

URL http://hal.inria.fr/hal-00794531

[10] G. Geymonat, F. Krasucki, D. Marini, M. Vidrascu, A domain decomposition method for a bonded structure, Math. Models Methods Appl. Sci. 8 (8) (1998) 1387-1402. doi:10.1142/S0218202598000652.

URL http://dx.doi.org/10.1142/S0218202598000652

[11] A.-L. Bessoud, F. Krasucki, M. Serpilli, Asymptotic Analysis of ShellLike Inclusions with High Rigidity, J. Elast 103 (2011) 153-172. doi:10.1007/s10659-010-9278-1.

[12] R. Abdelmoula, A. Leger, Local and global effects of small holes periodically distributed on a surface embedded in an axisymmetrical elastic medium, Eur. J. Mech. A Solids 24 (1) (2005) 89-109. doi:10.1016/j.euromechsol.2004.10.004.

URL http://dx.doi.org/10.1016/j.euromechsol.2004.10.004

[13] R. Abdelmoula, J.-J. Marigo, The effective behavior of a fiber bridged crack, J. Mech. Phys. Solids 48 (11) (2000) 2419-2444. doi:10.1016/S0022-5096(00)00003-X.

URL http://dx.doi.org/10.1016/S0022-5096(00)00003-X

[14] M. David, C. Pideri, J.-J. Marigo, Homogenized Interface Model Describing Inhomogeneities Located on a Surface, Journal of Elasticity (2011) accepté le 8/12/2011doi:10.1007/s10659-012-9382-5.

URL http://hal .archives-ouvertes.fr/hal-00655496 
[15] J.-J. Marigo, C. Pideri, The effective behavior of elastic bodies containing microcracks or microholes localized on a surface, International Journal of Damage Mechanics 20 (Special issue of ESMC2009) (2011) pages 1151-1177. doi:10.1177/1056789511406914.

URL http://hal-polytechnique . archives-ouvertes .fr/hal-00549546

[16] P. Le Tallec, Domain decomposition methods in computational mechanics, in: J. T. Oden (Ed.), Computational Mechanics Advances, Vol. 1 (2), North-Holland, 1994, pp. 121-220.

[17] J.-L. Lions, E. Magenes, Problèmes aux limites non homogènes et applications. Vol. 1, Travaux et Recherches Mathématiques, No. 17, Dunod, Paris, 1968.

[18] F.-X. Roux, Acceleration of the outer conjugate gradient by reorthogonalization for a domain decomposition method with lagrange multiplier, in: Proc. Third Intnl. Symp. Domain Decomposition methods, SIAM, 1990, pp. 314-321.

[19] J. Mandel, Balancing domain decomposition, Comm. Numer. Methods Engrg. 9 (3) (1993) 233-241. doi:10.1002/cnm.1640090307.

URL http://dx.doi.org/10.1002/cnm.1640090307

[20] P. Le Tallec, M. Vidrascu, Solving Large Scale Structural Problems on Parallel Computers using Domain Decomposition Techniques, m. papadrakakis Edition, J. Wiley, 1996, Ch. 3, pp. 49-82.

[21] C. Farhat, F.-X. Roux, Implicit parallel processing in structural mechanics, Comput. Mech. Adv. 2 (1) (1994) 124.

[22] A. Ralston, A first course in numerical analysis, McGraw-Hill Book Co., New York, 1965.

[23] P. G. Ciarlet, The finite element method for elliptic problems, Vol. 40 of Classics in Applied Mathematics, Society for Industrial and Applied Mathematics (SIAM), Philadelphia, PA, 2002, reprint of the 1978 original [North-Holland, Amsterdam; MR0520174 (58 \#25001)]. doi:10.1137/1.9780898719208.

URL http://dx.doi.org/10.1137/1.9780898719208 
[24] G. Grubb, Distributions and operators, Vol. 252 of Graduate Texts in Mathematics, Springer, New York, 2009. 